\title{
Identification of a bone marrow-derived epithelial-like population capable of repopulating injured mouse airway epithelium
}

\author{
Amy P. Wong, ${ }^{1}$ Armand Keating, ${ }^{2}$ Wei-Yang Lu, ${ }^{3}$ Pascal Duchesneau, ${ }^{1}$ \\ Xinghua Wang, ${ }^{2}$ Adrian Sacher, ${ }^{1}$ Jim Hu, ${ }^{4}$ and Thomas K. Waddell ${ }^{1}$
}

\begin{abstract}
${ }^{1}$ Latner Thoracic Surgery Research Laboratories, Toronto General Research Institute, and the McEwen Centre in Regenerative Medicine, Toronto, Ontario, Canada. 2Princess Margaret Hospital Ontario Cancer Institute, Faculty of Medicine and Institute for Biomaterials and Biomedical Engineering, University of Toronto, Toronto, Ontario, Canada. ${ }^{3}$ Sunnybrook Health Sciences Centre, Toronto, Ontario, Canada. 4Programme in Physiology and Experimental Medicine, Hospital for Sick Children, Toronto, Ontario, Canada.
\end{abstract}

\begin{abstract}
The bone marrow compartment is enriched in stem and progenitor cells, and an unidentified subpopulation of these cells can contribute to lung epithelial repair. Here we identify this subpopulation and quantitate its relative contribution to injured airway epithelium. A subpopulation of adherent human and murine bone marrow cells that expresses Clara cell secretory protein (CCSP) was identified using flow cytometry. When cultured at the air-liquid interface in ex vivo cultures, $\mathrm{Ccsp}^{+}$cells expressed type I and type II alveolar markers as well as basal cell markers and active epithelial sodium channels. $\operatorname{Ccsp}^{+}$cells preferentially homed to naphthalenedamaged airways when delivered transtracheally or intravenously, with the former being more efficient than the latter. Interestingly, naphthalene-induced lung damage transiently increased Ccsp expression in bone marrow and peripheral circulation. Furthermore, lethally irradiated Ccsp-null mice that received tagged wild-type bone marrow contained donor-derived epithelium in both normal and naphthalene-damaged airways. This study therefore identifies what we believe to be a newly discovered cell in the bone marrow that might have airway reconstitution potential in the context of cell-based therapies for lung disease. Additionally, these data could reconcile previous controversies regarding the contribution of bone marrow to lung regeneration.
\end{abstract}

\section{Introduction}

Adult bone marrow contains a variety of stem cell populations that can be recruited to injured tissues. Although controversial, studies have suggested the possible involvement of bone marrowderived hematopoietic, mesenchymal, or Hoechst-effluxing side population cells in lung repair following injury (1-6). Moreover, in vitro studies have shown that bone marrow cells (BMCs) cultured either alone or in coculture with epithelium can express epithelial functional properties (tight junction formation and the CFTRmediated chloride ion transport) (7), which further suggests a potential therapeutic use of BMCs in treatment of pulmonary diseases. More recent studies employing transgenic animal models and robust analytical methods to assess pulmonary engraftment of BMCs have shown only very low levels of involvement of bone marrow in lung regeneration $(8,9)$. Although variations in technical and analytical methods used to assess BMC engraftment and/or transdifferentiation may explain these discrepant observations, a likely explanation is uncertainty regarding the specific subpopulation of BMCs that have pulmonary engraftment and regenerative potential.

We report here what we believe is a newly discovered population of cells in mouse and human bone marrow that express Clara cell secretory protein (CCSP), a marker of airway progenitor (10,

Conflict of interest: The authors have declared that no conflict of interest exists. Nonstandard abbreviations used: ALI, air-liquid interface; AQP5; aquaporin 5; $\mathrm{BMC}$, bone marrow cell; Ccsp, Clara cell secretory protein; $\mathrm{ENaC}$, epithelial sodium channel; K5, cytokeratin-5; MSC, mesenchymal stem cell; proSpc, prosurfactant protein-C; SSEA, stage-specific embryonic antigen.

Citation for this article: J. Clin. Invest. 119:336-348 (2009). doi:10.1172/JCI36882.
11) and stem cells (12). These cells were isolated, proliferated in culture, and increased in bone marrow and peripheral blood in response to airway injury. The majority of $\mathrm{Ccsp}^{+}$cells expressed CD45 and mesenchymal markers CD73, CD90, CD105. These cells demonstrated the ability to home to the lung following either injection of cultured cells or bone marrow transplantation. They differentiated into multiple epithelial cell lineages, including both airway and alveolar lineages, following air-liquid interface (ALI) culture. This cell population might be used in cell replacement strategies for epithelial diseases.

\section{Results}

A Ccsp-expressing population exists in the bone marrow of mice and bumans

BMCs were previously identified in the lung expressing Ccsp as early as 1 day after intratracheal administration (13). This led us to examine the starting BMC population. A significant proportion of the adherent cells expressed Ccsp. Moreover, freshly isolated bone marrow also contained a small percentage of cells that expressed Ccsp on the cell surface (Figure 1A, arrow). Multiple anti-Ccsp primary antibodies showed similar results (data not shown). The population of BMCs that expressed Ccsp $(1.9 \% \pm 0.1 \%)$ expanded in culture up to $25.83 \% \pm 9.47 \%$ after 7 days (Figure 1B). Western blotting of freshly isolated and cultured BMCs confirmed the existence of an 11-kDa protein (as expected for Ccsp) in BMCs (Figure 1C). The bone marrow of Ccsp knockout mice did not show any $\mathrm{Ccsp}^{+}$cells (Figure 1D). Consistently, real-time PCR detected the expression of Ccsp mRNA in freshly isolated and cultured BMCs of wild-type but not Ccsp knock- 
A

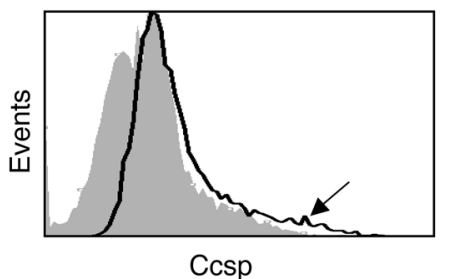

D

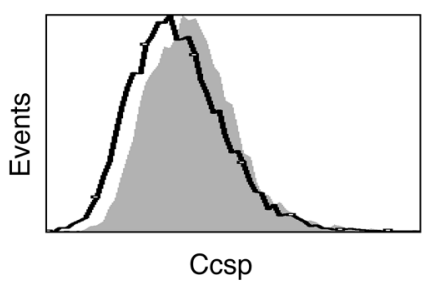

G

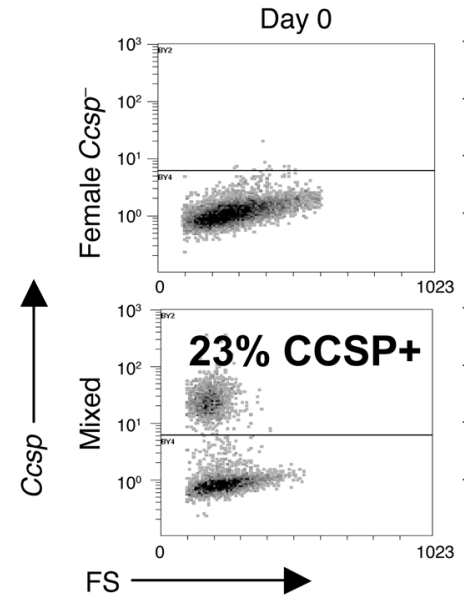

B

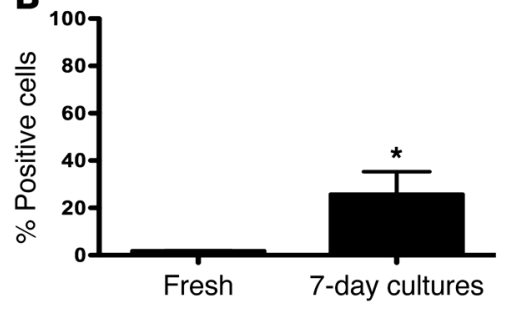

E

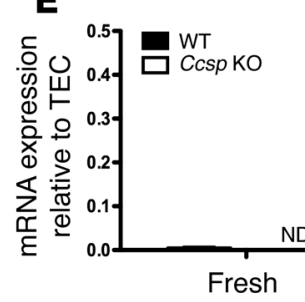

7-day cultures
C

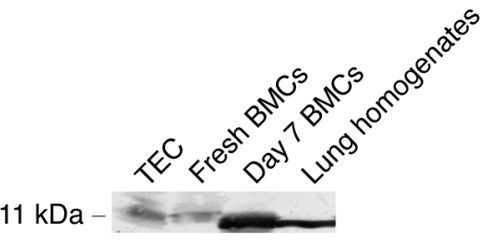

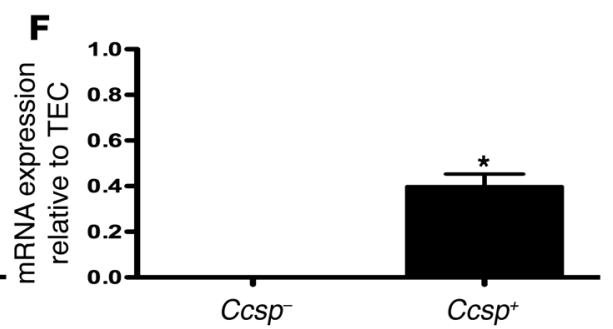
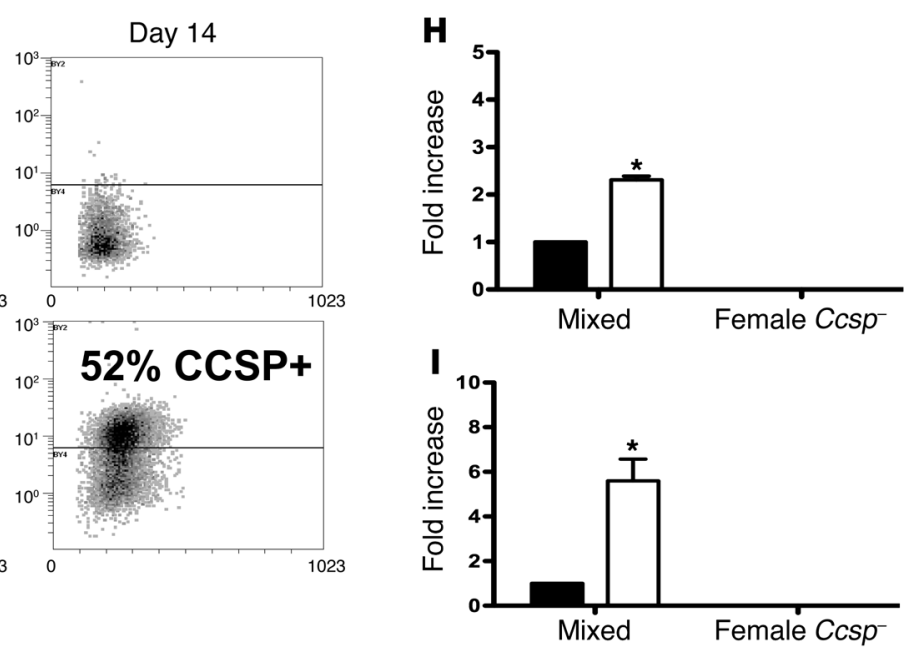

Figure 1

Identification of a bone marrow Ccsp-expressing population. (A) Representative flow histograms (solid black line) of freshly isolated BMCs compared with isotype staining (gray histogram) show a small population (arrow) of BMC-expressing Ccsps. (B) Freshly isolated bone marrow contained approximately $2 \% \mathrm{Ccsp}^{+}$cells, which expanded in culture after 7 days to approximately $25 \%$ Ccsp ${ }^{+}\left({ }^{*} P<0.001\right)$. $n=8$ per group. (C) Western blot analysis confirmed the presence of an $11-\mathrm{kDa}$ band (tracheal epithelial cells [TECs] were loaded with $10 \%$ of the protein used for BMCs and lung homogenates) and in BMCs freshly isolated or cultured for 7 days. (D) No Ccsp+ BMC population was detected in Ccsp knockout mice. Solid black line indicates Ccsp antibody; gray histogram indicates isotype staining. (E) Real-time PCR confirmed the presence of a Ccsp gene transcript in freshly isolated and cultured BMCs of wild-type but not Ccsp knockout animals. ND, not detectable. Gapdh was used as a housekeeping gene for normalization of expression levels. Each bar represents normalized levels relative to tracheal epithelial cells. $n=4$ per group. (F) Real-time PCR detected Ccsp gene expression in Ccsp ${ }^{+}$but not Ccsp- cells. $n=12$ per group; ${ }^{\star} P<0.01$ compared with freshly isolated cells. (G) Female Ccsp ${ }^{-}$cells cultured alone on plastic for 14 days showed no detectable Ccsp ${ }^{+}$cells, while a mixed population showed an increase in Ccsp ${ }^{+}$cells after 14 days in culture. (H) Real-time PCR showed a 2.5-fold increase in Sry mRNA after 14 days in culture in the mixed population. (I) The increase in Ccsp $p^{+}$cell number corresponded to an increase in Ccsp mRNA. Each bar represents mean \pm SEM $(n \geq 3)$. ${ }^{*} P<0.01$ compared with $C c s p-$ cells.

out animals (Figure 1E). Because Ccsp is widely known as an intracellular or secreted protein, the finding of detectable surface staining was surprising. Confocal microscopy of cultured BMCs showed that Ccsp was also seen inside the cell (data not shown). FACS sorting of cells confirmed that $\mathrm{Ccsp}^{+} \mathrm{BMCs}$ had appreciable levels of Ccsp mRNA, while Ccsp- cells did not (Figure 1F). A small $\mathrm{CCSP}^{+} \mathrm{BMC}$ population was also found in healthy human volunteers (Supplemental Figure 1, A-E; supplemental material available online with this article; doi:10.1172/JCI36882DS1). Real-time PCR showed detectable levels of CCSP mRNA in 4 of 5 human bone marrow samples (Supplemental Figure 1F). Ccsp $\mathrm{P}^{+}$ cells could also be found in low levels in the peripheral lymph nodes, but not in the spleen (data not shown).

The increased proportion of $\mathrm{Ccsp}^{+}$cells seen in culture might be due to conversion of $\mathrm{Ccsp}^{-}$to $\mathrm{Ccsp}^{+}$cells or to differences in survival or proliferation of these cells. Cultured BMCs were sorted by FACS and examined for Ccsp expression immediately and after 14 days in culture. Ccsp ${ }^{-}$cells did not give rise to $\mathrm{Ccsp}^{+}$cells. Because $\mathrm{Ccsp}^{+}$cells did not grow well in culture alone, female Ccsp ${ }^{-}$and male Ccsp ${ }^{+}$cells were mixed together at a 4:1 female/male ratio. 
research article
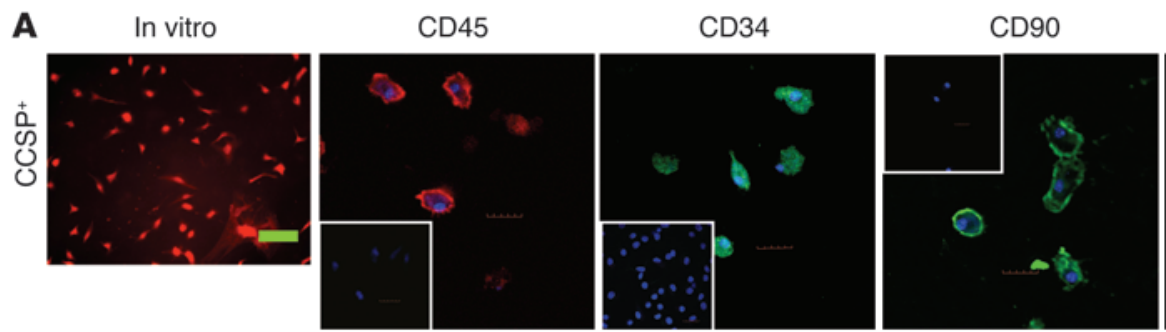

CD105

CD73
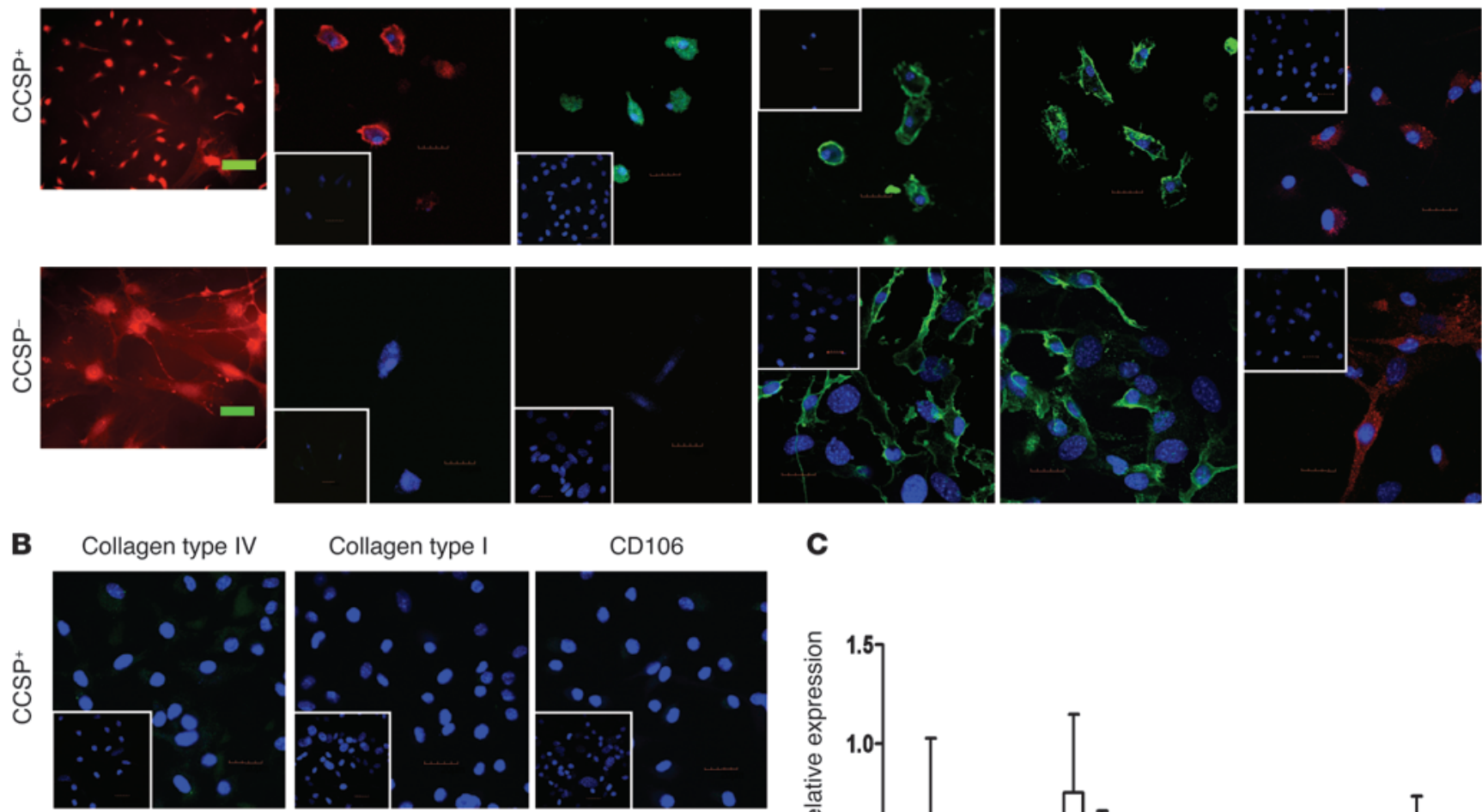

Collagen type I

CD106

C

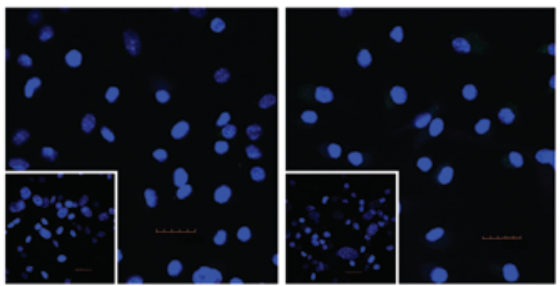

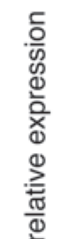
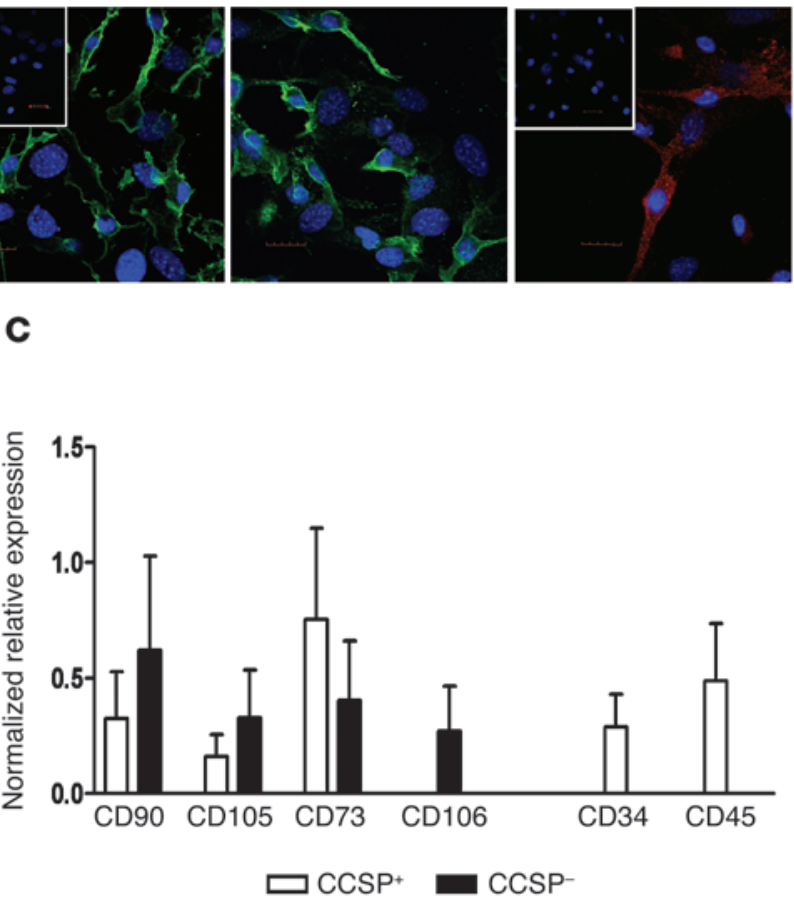

D
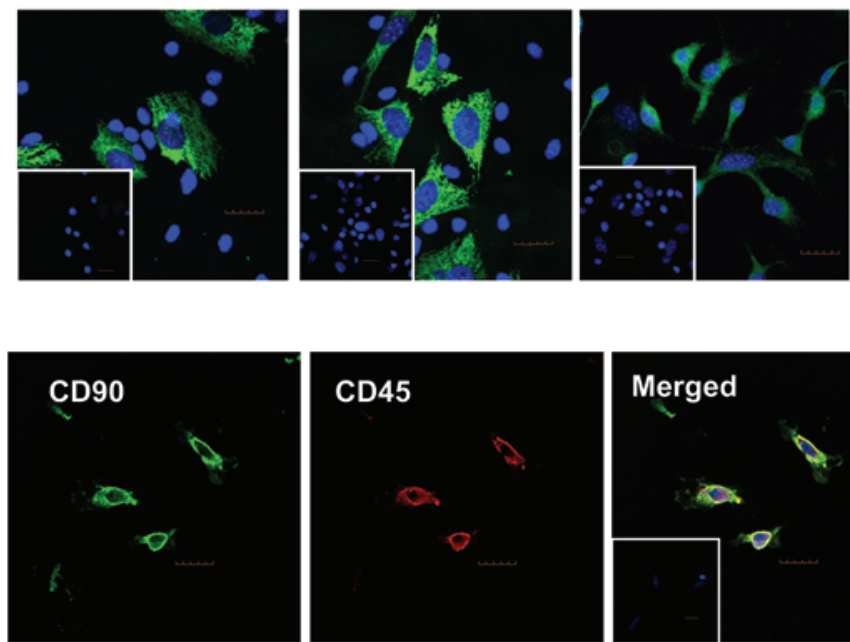

$\square \mathrm{CCSP}^{+}-\mathrm{CCSP}^{-}$
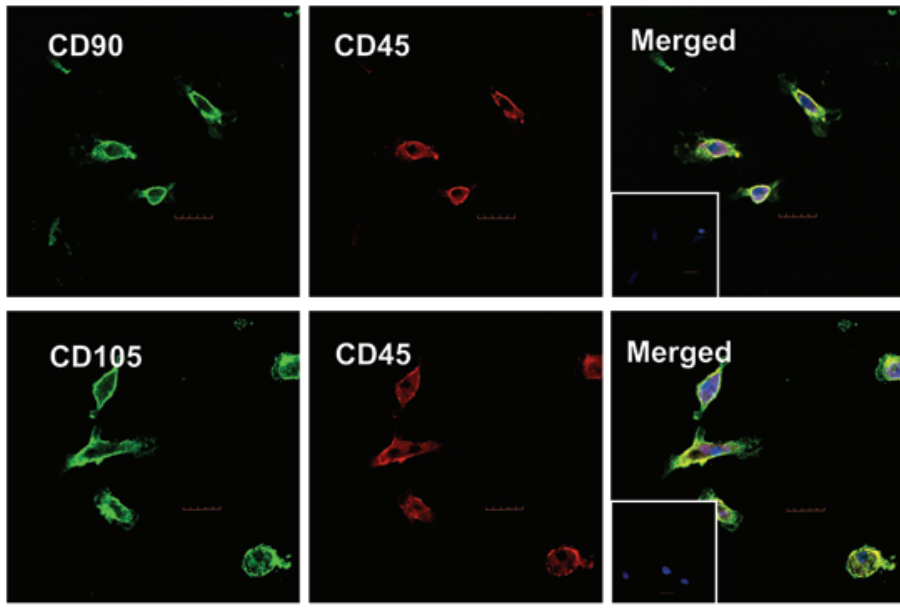

Red Channel
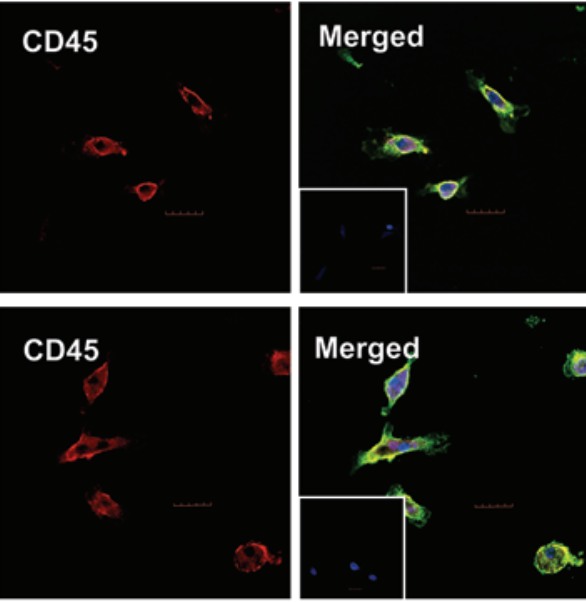

Green Channel

20 


\section{Figure 2}

Characterization of the Ccsp ${ }^{+}$BMC population. (A) Morphologically, $\mathrm{Ccsp}^{+}$and $\mathrm{Ccsp}^{-}$cells were distinguishable in vitro. Ccsp ${ }^{+}$cells were smaller, rounder cells, while Ccsp- cells were larger, with more cytoplasmic extensions. Ccsp ${ }^{+}$cells expressed pan-hematopoietic marker CD45 and progenitor marker CD34. Both Ccsp ${ }^{+}$and Ccsp- cells expressed the MSC markers CD73, CD90, and CD105. (B) Ccsp ${ }^{+}$ cells did not express the MSC markers CD106, type I collagen, or type IV collagen. Some Ccsp- cells expressed CD106, type IV collagen, and type I collagen. Insets are representative isotype staining controls. (C) Real-time PCR showed gene expression of some MSC markers (CD90, CD105, CD73) in Ccsp+ cells but not CD106. Hematopoietic genes CD45 and CD34 were also detected in Ccsp ${ }^{+}$ cells. Conversely, Ccsp- cells expressed all mesenchymal stromal cell genes but no hematopoietic genes. GAPDH was used as housekeeping gene for normalization of expression levels. Each bar represents normalized relative levels compared with MSCs (bone marrow nonadherent cells for CD45 and CD34). $n=4$ sets of cells from 4 different animals. (D) Most Ccsp ${ }^{+}$cells coexpressed both hematopoietic (CD45) and mesenchymal markers (CD90 and CD105). Control staining with CD45-PE (red) and secondary antibody specific for CD90/CD105 showed no cross-reactivity of the secondary antibody binding to CD45 primary antibody (no fluorescence in the green channel). Mixed isotypes were used as a control for nonspecificity of the primary antibody (inset). Hoechst counterstain was used to visualize nuclei (blue). All scale bars: $20 \mu \mathrm{m}$. Original magnification, $\times 20$.

$\mathrm{Ccsp}^{+}$cells almost doubled after 14 days in culture (Figure 1G). Assessment of SRY (male sex-determining gene) genomic DNA and Ccsp mRNA using real-time PCR showed a 2.5-fold increase in SRY DNA (Figure 1H) with a concomitant increase in Ccsp mRNA (Figure 1I), suggesting $\mathrm{Ccsp}^{+}$cell proliferation as the cause of the increase in $\mathrm{Ccsp}^{+}$cells.

\section{Characterization of $\mathrm{Ccsp}^{+} \mathrm{BMCs}$}

Morphologically, Ccsp $\mathrm{P}^{+}$cells were small $(\sim 5-10 \mu \mathrm{m})$, round cells, and a few had small cytoplasmic extensions. In contrast, Ccsp $\mathrm{P}^{-}$cells (>10 $\mu \mathrm{m})$ ranged from very large cells with a lot of cytoplasm to medium-sized cells with large cytoplasmic extensions (Figure 2A).

Phenotypic characterization of the $\operatorname{Ccsp}^{+}$and $\operatorname{Ccsp}^{-}$cells show that in contrast to Ccsp- cells and in spite of their ability to adhere, the majority of Ccsp ${ }^{+}$cells expressed CD45 and CD34. Ccsp ${ }^{+}$cells were negative for CD11b (data not shown). The majority of the $\mathrm{Ccsp}^{+}$cells also expressed the mesenchymal markers CD73, CD90, and CD105 but not CD106, collagen type I or collagen type IV (Figure 2B). Mesenchymal stem cell (MSC) markers were observed in some but not all Ccsp- cells. Mouse MSCs were used as positive controls for MSC markers (Supplemental Figure 2). Ccsp ${ }^{+}$cells did not express von Willebrand factor or vimentin (data not shown). Real-time PCR confirmed mRNA expression of the mesenchymal and hematopoietic genes in $\mathrm{Ccsp}^{+}$cells (Figure 2C). To determine whether the same $\mathrm{Ccsp}^{+}$cells expressing MSC markers were also expressing hematopoietic markers, double immunofluorescence staining was performed. The same CD90- or CD105-expressing $\mathrm{Ccsp}^{+}$coexpressed CD45 (Figure 2D). Appropriate isotype controls were used for each specific stain. To eliminate the possibility of cross-reactivity of the secondary antibody, $\mathrm{Ccsp}^{+}$cells were stained with primary antibody-labeled CD45-PE followed by goat anti-rat Alexa Fluor secondary antibody. No fluorescence signal was observed in the green channel of these stained cells, suggesting the secondary antibody specific for CD90 and CD105 did not bind to CD45-PE primary antibody or nonspecific surface antigens and no "bleed-through" from the CD45 antibody. As negative controls, MSCs and a mouse fibroblast cell line (NIH3T3) were doublestained for CD90 or CD105 and CD45 (Supplemental Figure 3).

\section{Multi-epithelial lineage differentiation of $C c s p^{+}$BMCs}

To determine whether $\mathrm{Ccsp}^{+}$cells could give rise to other epithelial cell lineages, cells were cultured in ALI conditions. Airway epithelial cells were used as positive controls for both the ALI cultures and immunofluorescence staining. Representative nonspecific immunoglobin (IgG) staining of isolated airway epithelial cells is shown in Figure 3A. Nonspecific IgG staining on $\mathrm{Ccsp}^{+}$and $\mathrm{Ccsp}^{-}$cells were also done as negative controls (data not shown). To ensure specificity of colocalized fluorescent signals, control tracheal epithelial cells were stained with one specific primary antibody and excited with the appropriate excitation $(350,488,532$, or $633 \mathrm{~nm})$ lasers, then emission wavelength images were monitored for the specific fluorophore and the other channels used in the experimental groups (Figure 3B). Background fluorescence was low in the other fluorophore channels, suggesting that the observed colocalization was not an artifact of bleed-though between the fluorescence channels. Most $\mathrm{Ccsp}^{+}$cells cultured under ALI for 4 weeks lose Ccsp expression (Supplemental Figure 4) and gain other epithelial characteristics such as protein expression of prosurfactant protein-C (proSpc) (type II alveolar cell), cytokeratin-14 (K14; basal cell), and/or aquaporin 5 (AQP5; type I alveolar cell). $\mathrm{Ccsp}^{+}$cells gave rise to proSpc ${ }^{+}$and $\mathrm{AQP5}^{+}$cells. A large number of cells were positive for both SPC and other epithelial markers (Figure 3C). Ccsp ${ }^{-}$cells only gave rise to rare $\mathrm{SPC}^{+}$cells (data not shown). In addition, $\mathrm{Ccsp}^{+}$cells gave rise to few $\mathrm{K}^{+} 4^{+}$, acetylated

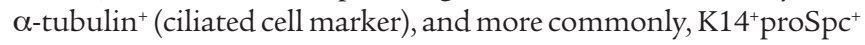
and acetylated $\alpha$-tubulin ${ }^{+}$proSpc $^{+}$cells (Figure 3C). Furthermore, all ALI-cultured Ccsp ${ }^{+}$cells lost the hematopoietic marker CD45 and mesenchymal markers CD90/CD105 (data not shown). Transmission electron microscopy of these cells did not show ultrastructural features of clearly defined lamellar bodies and cilia (data not shown), suggesting that at least in vitro, these cells do not have complete epithelial differentiation potential.

Immediately after FACS sorting, $\mathrm{Ccsp}^{+}$cells did not express other epithelial genes except very low levels of proSpc, while Ccsp ${ }^{-}$cells did not express any epithelial genes (Supplemental Figure 5). After ALI culture, $\mathrm{Ccsp}^{+}$cells expressed other genes typical of mature epithelial cells, including AQP5, proSpc, K5, K14, K18, and Ccsp, while Ccsp- cells did not (Figure $3 \mathrm{D}$ ). Ccsp $\mathrm{C}^{-}$cells did have a very faint PCR signal for proSpc (not visible in Figure 3 due to the scale).

\section{Regenerative potential of Ccsp ${ }^{+}$cells}

$C c s p^{+} B M C s$ are differentially retained in injured airways. To determine whether the $\mathrm{Ccsp}^{+} \mathrm{BMCs}$ are more efficiently retained in the lung, sorted $\mathrm{Ccsp}^{+}$and $\mathrm{Ccsp}^{-}$cells labeled with the commercial red fluorescent marker 5-(and-6)-([(4-chloromethyl)benzoyl $]$ amino) tetramethylrhodamine (CMTMR) were injected transtracheally or intravenously into naphthalene-injured recipients as previously described (13). Four days later, the lungs were harvested. Donor BMCs were detected by fluorescence microscopy for CMTMR (left lung), and retention was quantified by real-time PCR for the Sry gene (right lung). More Ccsp ${ }^{+}$cells (either retention or proliferation) were found in injured airway epithelium compared with Ccsp- cells when cells were delivered transtracheally to the airways (Figure 4A). Under high-power magnification, most Ccsp ${ }^{+}$cells incorporated into the epithelium, whereas $\mathrm{Ccsp}^{-}$cells remained on 
A
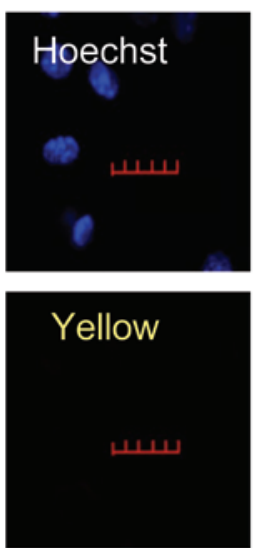

B
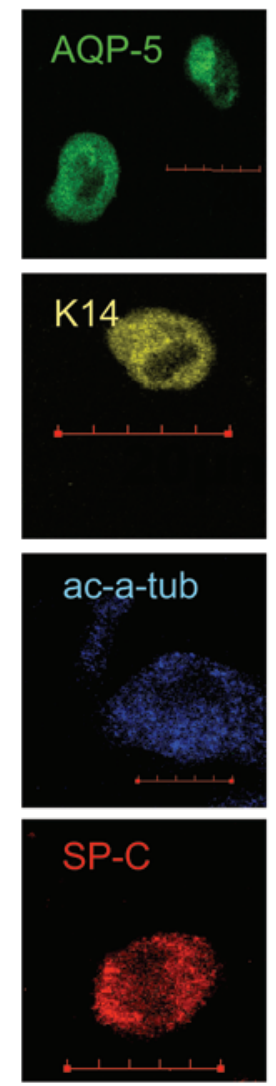
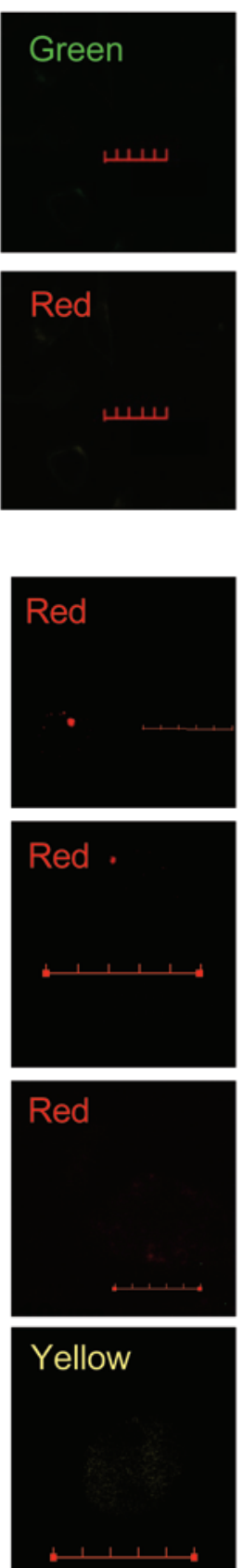

C
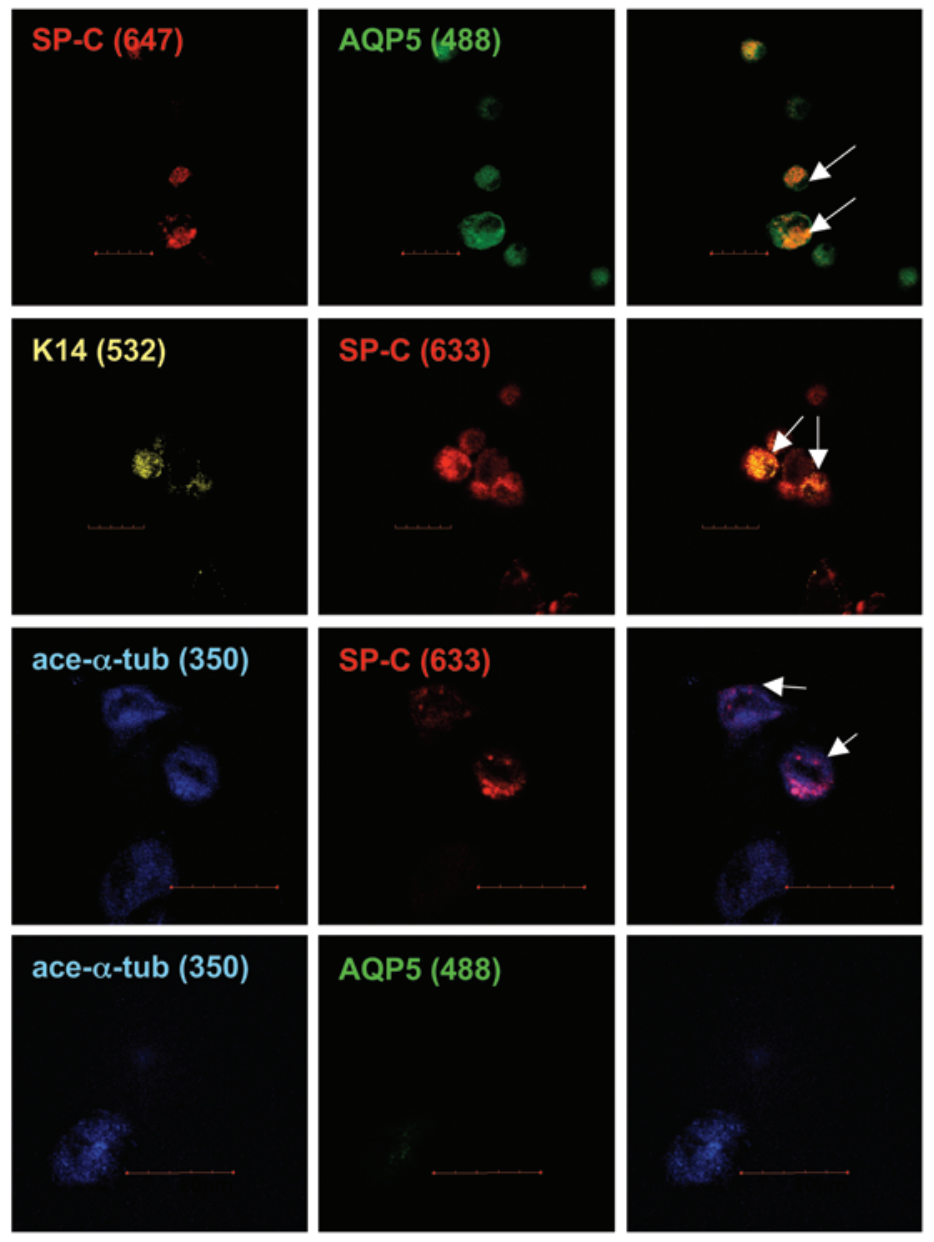

D

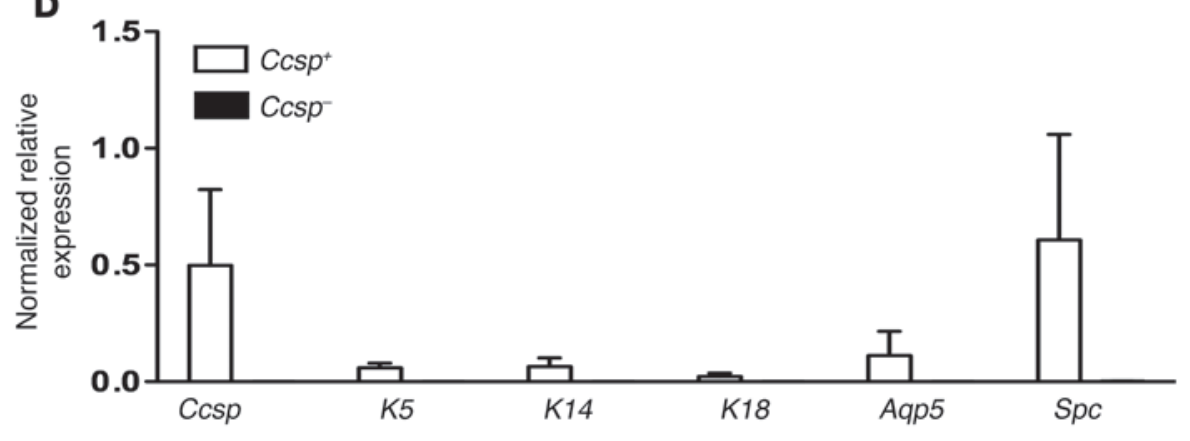

Figure 3

Epithelial multilineage differentiation of Ccsp+ BMCs. (A) Isotype mix control staining of airway epithelial cells showed no cross-reactivity of the antibodies. (B) Control staining of isolated airway epithelial cells for each antibody. Bleed-through of signal is visualized in depicted representative images. Scale bars: $20 \mu \mathrm{m}$. (C) After 4 weeks in ALI culture, Ccsp ${ }^{+}$cells gave rise to a multitude of epithelial lineage cells including proSpc ${ }^{+}$,

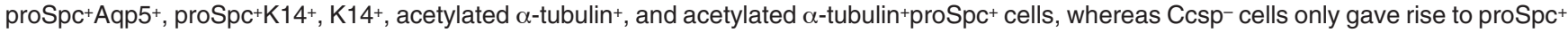
cells. White arrows point to double-positive cells. Hoechst counterstain was used to visualize nuclei (blue). Numbers in parentheses indicate the fluorescent emission wavelengths for the stains. Scale bars: $10 \mu \mathrm{m}$. Original magnification, $\times 40$. (D) Real-time PCR confirmed expression of various epithelial genes in ALI-cultured Ccsp ${ }^{+}$cells. Ccsp ${ }^{+}$cells expressed various epithelial genes including Ccsp, K5, K14, K18, Aqp5, and proSpc, whereas Ccsp- cells only expressed proSpc. Gapd $h$ was used as housekeeping gene for normalization of expression levels. Ccspsorted cells were cultured in a mixture of bone marrow- and epithelial cell-specific media and in ALI conditions to induce epithelial differentiation. Each bar represents normalized relative levels compared with tracheal epithelial cells. $n=4$ sets of cells from 4 different animals. 
A
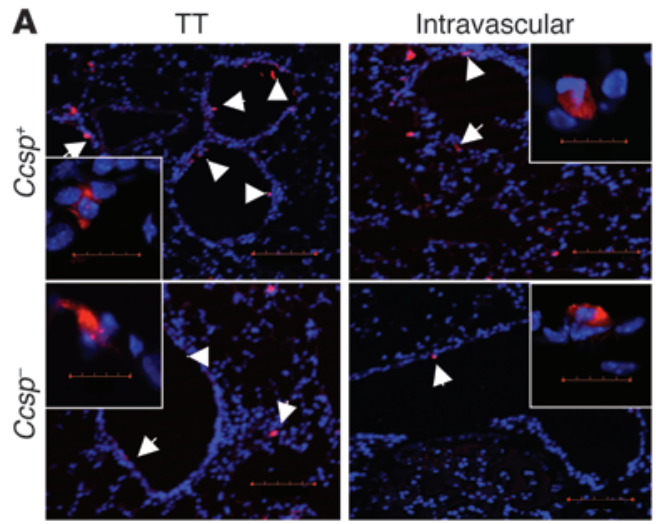

C

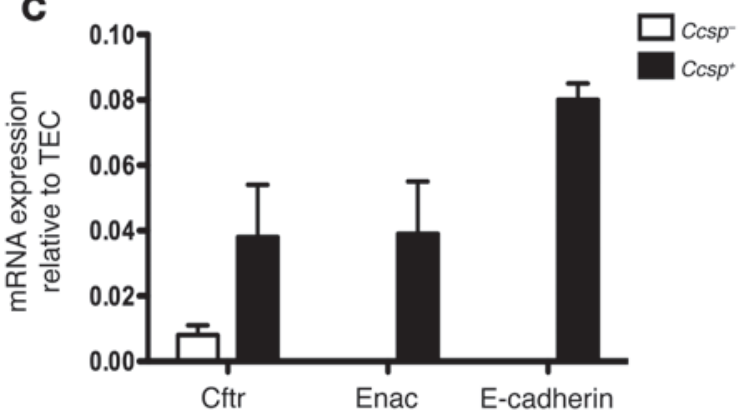

E

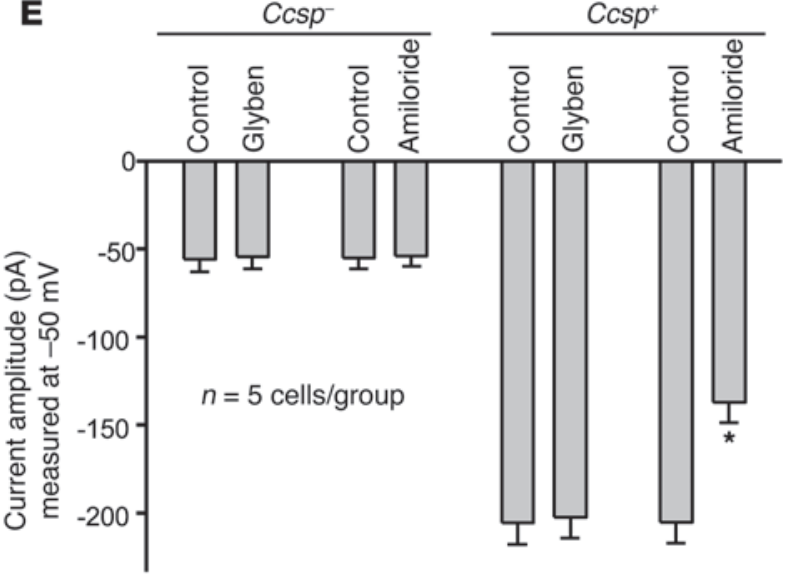

B

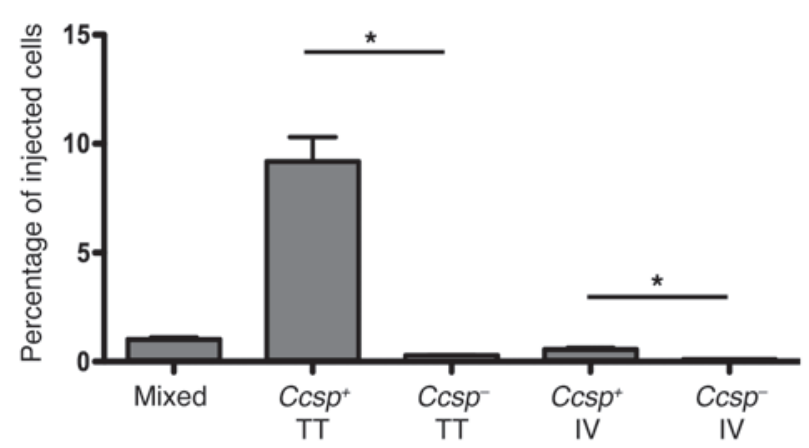

D
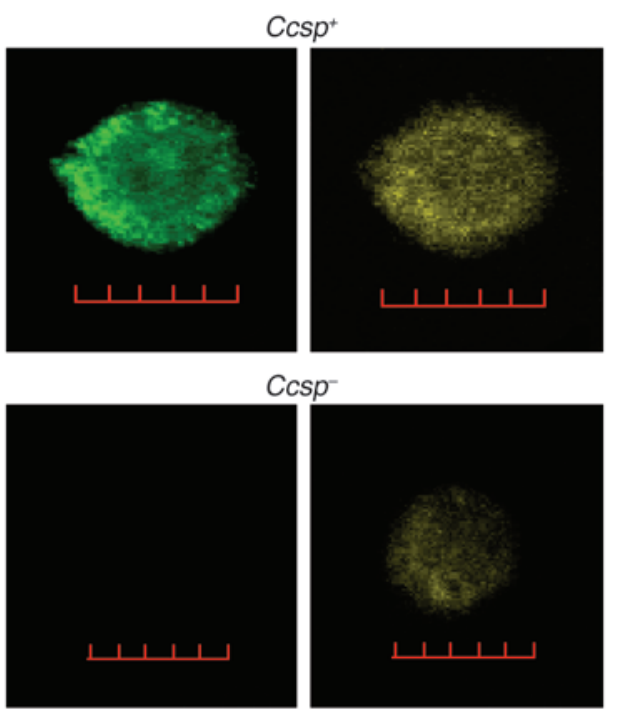

Figure 4

Regenerative potential of Ccsp+ BMCs. (A) Both transtracheal (TT) and intravenous (IV) delivery of Ccsp+ or Ccsp- BMCs (red) after naphthalene-induced airway injury show greater airway retention of $\mathrm{Ccsp}^{+}$cells than $\mathrm{Ccsp}^{-}$cells. Scale bars: $100 \mu \mathrm{m}$. Original magnification, $\times 20$. Insets show a representative cell (scale bars: $20 \mu \mathrm{m}$; original magnification, $\times 120$ ). (B) Of the $10^{6}$ cells injected, transtracheal delivery of Ccsp ${ }^{+}$ cells showed the most cell retention ( $9 \%)$ measured 4 days after cell injection. $n=4$ mice per group. ${ }^{*} P<0.01$ compared with Ccsp- cells. (C) Ion channel genes Cftr and Enac and the tight junction protein E-cadherin were detected by real-time RT-PCR of Ccsp ${ }^{+}$cells. Ccsp ${ }^{+}$BMC levels of Cftr and Enac corresponded to $4 \%$ of the levels detected in airway epithelial cells, while E-cadherin levels corresponded to $8 \%$. Low but detectable levels of Cftr (<1\%) were also detected in Ccsp- cells. $n=12$ mice per group. (D) Ccsp cells coexpressed Cftr (green) and Enac (red) proteins. Hoechst counterstain was used to visualize nuclei (blue). Scale bars: $10 \mu \mathrm{m}$. (E) Perforated patch clamp recordings show the total conductance in $\mathrm{Ccsp}^{+}$cells was larger than in $\mathrm{Ccsp}^{-}$cells. Treating Ccsp- cells with glybenclamide and amiloride did not change their total conductance. Application of glybenclamide did not alter the total conductance of $\mathrm{Ccsp}^{+}$cells, but amiloride significantly suppressed the total conductance, suggesting that $\mathrm{Ccsp}^{+}$cells express functional Enac properties. $n=5$ cells per group. ${ }^{\star} P<0.01$ compared with control.

the airway epithelium. Of the $10^{6} \mathrm{BMCs}$ injected (to both lungs), about $10 \%$ of the $\mathrm{Ccsp}^{+}$cells were detected in the right lung after transtracheal delivery compared with about $1 \%$ after intravenous delivery. Retention in the lung was far greater for $\mathrm{Ccsp}^{+}$than $\mathrm{Ccsp}^{-}$ cells $\left({ }^{*} P<0.01\right.$; Figure $\left.4 \mathrm{~B}\right)$.
ALI-cultured Ccsp ${ }^{+}$BMCs express epithelial ion channel proteins and have some epithelial ion transport properties. mRNAs for epithelial ion channels (epithelial sodium channel [Enac] and Cftr) and the tight junction protein E-cadherin were detected in $\mathrm{Ccsp}^{+} \mathrm{BMCs}$ but not $\mathrm{Ccsp}^{-}$ cells by real-time PCR (Figure 4C). Double immunofluorescence 


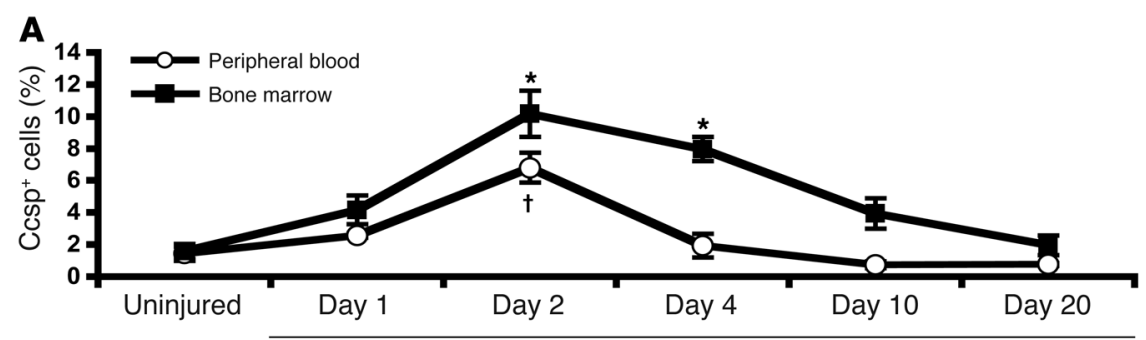

Airway injured

B

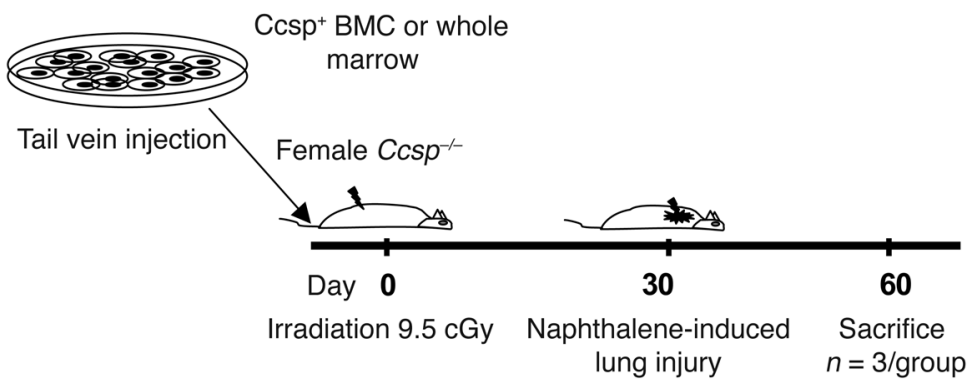

C

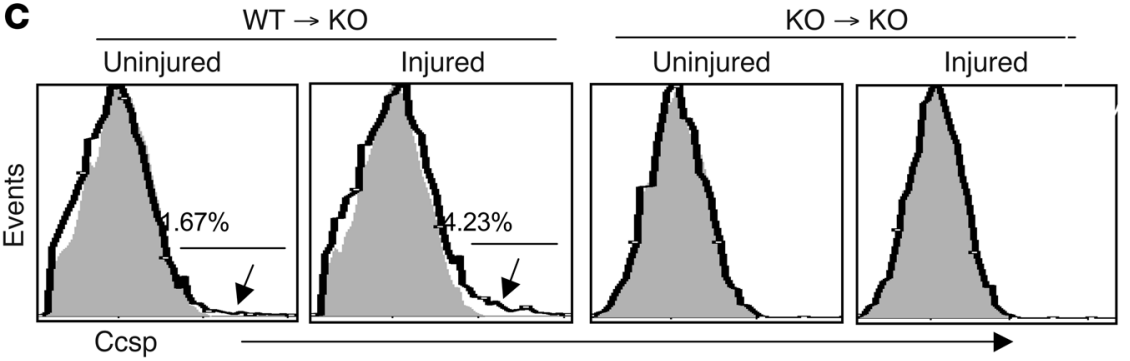

D

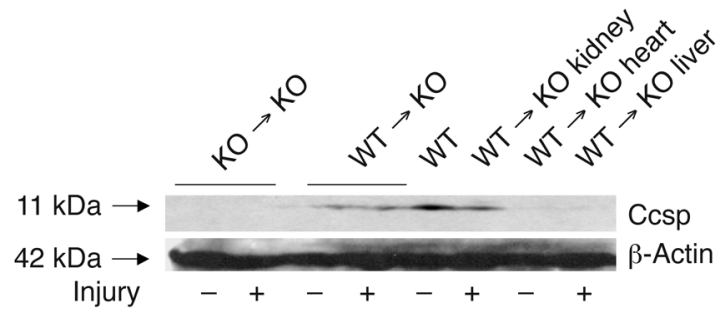

\section{Figure 5}

Endogenous bone marrow Ccsp ${ }^{+}$cells can respond to airway injury. Ccsp protein was detected in the bone marrow and lungs of bone marrow-transplanted Ccsp knockout animals. (A) Bone marrow and peripheral blood of mice after naphthalene-induced airway injury showed a transient increase in $\mathrm{Ccsp}^{+}$cells, peaking at 2 days of airway injury and declining to baseline levels by 20 days. $n=4$ mice per group. ${ }^{*} P<0.01 \mathrm{com}$ pared with uninjured peripheral blood group; ${ }^{\dagger} P<0.05$ compared with uninjured bone marrow group. (B) Strategy of the bone marrow transplants. (C) Flow cytometry of bone marrow transplantation of wild-type whole marrow showed existence of Ccsp ${ }^{+}$ BMCs, which increased with airway injury. No detectable Ccsp ${ }^{+}$BMCs was found in mice that received Ccsp knockout marrow with or without airway injury. Percentages indicate percentage of cells above background fluorescence. Arrows indicate part of histogram at which cells were positive for Ccsp. (D) Western blot confirmed the presence of the $11-\mathrm{kDa}$ band corresponding to the Ccsp protein in the lungs and kidneys of mice that received wild-type but not Ccsp knockout marrow. staining for CFTR and $\mathrm{ENaC}$ showed that $\mathrm{Ccsp}^{+}$cells expressed both proteins (Figure 4D), while most $\mathrm{Ccsp}^{-}$cells expressed neither. Rare Ccsp- cells expressed small levels of CFTR but not ENaC (Figure 4D). Perforated patch clamp recordings were carried out to examine functional CFTR and ENaC activity. We found that the total conductance in $\mathrm{Ccsp}^{+}$cells was larger than that in Ccsp $\mathrm{P}^{-}$cells (Figure 4E; measured at $-50 \mathrm{mV}, \mathrm{Ccsp}^{+}:-205 \pm 12 \mathrm{pA}, n=5$ cells; Ccsp $:-54 \pm 8$ pA, $n=5$ cells; $P<0.005)$. Application of forskolin and glybenclamide did not alter the total conductance of Ccsp $\mathrm{P}^{+}$, suggesting that CFTR is not functional. Amiloride significantly suppressed the total conductance (Figure 4E), suggesting that $\mathrm{ENaC}$ is indeed functional in these cells. Consistent with the lack of ion channel expression in Ccsp- cells, treating Ccsp $\mathrm{P}^{-}$cells with glybenclamide and amiloride did not change their conductance.

\section{Endogenous role of Ccsp ${ }^{+}$BMCs in repair of lung injury}

Naphthalene-induced lung injury transiently increases the Ccsp ${ }^{+} B M C$ population in the bone marrow and peripheral blood. To explore whether endogenous $\mathrm{Ccsp}^{+} \mathrm{BMCs}$ may be involved in lung repair, we assessed $\mathrm{Ccsp}^{+}$cells in the bone marrow and peripheral blood after naphthalene-induced lung injury. In contrast to the lung, where naphthalene depletes $\operatorname{Ccsp}^{+}$cells $(13,14)$, the number of Ccsp ${ }^{+}$cells in marrow and peripheral blood increased as early as 2 hours after naphthalene injury and peaked at 2 days after injury (Figure $5 \mathrm{~A}$ ). The number of $\mathrm{Ccsp}^{+}$cells remained elevated for 4 days and returned to near baseline by 20 days. Unlike lung epithelium, BMCs did not express CYP2F2, the P450 isoenzyme involved in generation of toxic metabolites leading to lung epithelial cell death (data not shown).

Endogenous $C c s p^{+}$BMCs can contribute to lung epithelium regeneration. To determine their endogenous role, bone marrow (either whole marrow or $\mathrm{Ccsp}^{+}$cells $\left[3 \times 10^{5}\right.$ cells per mouse] from wild-type mice mixed with whole marrow $\left[2 \times 10^{7}\right.$ cells] from Ccsp knockout mice) was injected into female Ccsp knockout recipients after lethal irradiation (Figure 5B). As controls, whole marrow was transplanted from Ccsp knockout mice. Thirty days after transplantation, half the mice from each group received an injection of 

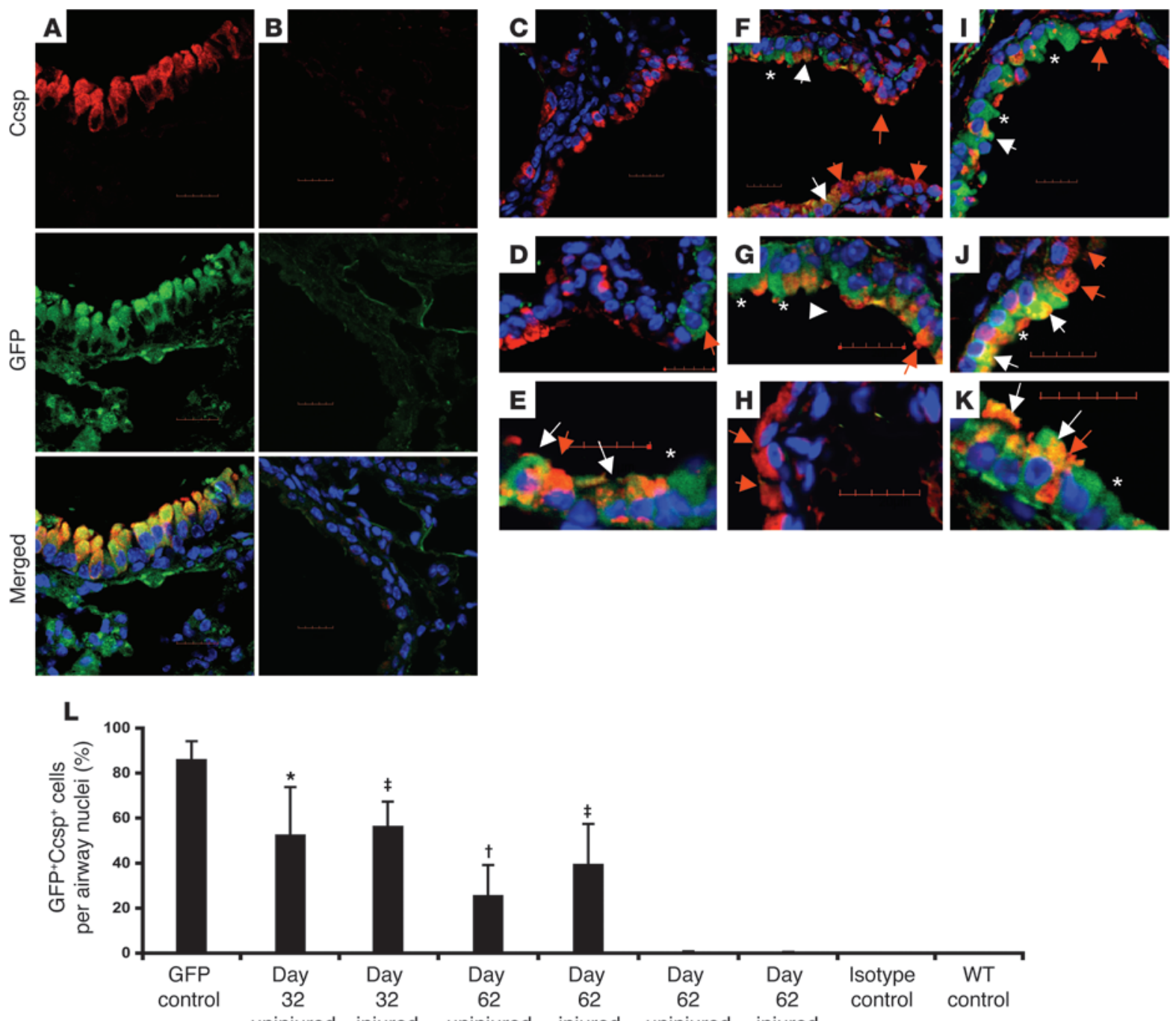

uninjured injured uninjured injured uninjured injured

KO recipient

WT recipient

\section{Figure 6}

Endogenous bone marrow $\mathrm{Ccsp}^{+}$cells can repopulate the airway epithelium. (A-K) Representative double immunofluorescence staining for GFP (green) and Ccsp (red) of lung sections from bone marrow transplant recipient mice that received Ccsp + Sca- $1+$ cells from GFP+ donors. Asterisks indicate a GFP+ cell that was Ccsp-. White arrows point to donor-derived Ccsp ${ }^{+}$cells. Red arrows point to Ccsp ${ }^{+}$cells that were not donor derived. (A) GFP+ (positive control) lung. (B) Isotype staining of lung from bone marrow transplant recipient. (C-E) Bone marrow transplant recipients had a wild-type background. (C) Low-power image. (D and E) High-power images. (F-H) Bone marrow transplant recipients were $\mathrm{Cssp}^{-1}$. Sixty days following bone marrow transplant without naphthalene. $(\mathbf{F})$ Low-power image. (G and $\left.\mathbf{H}\right)$ High-power image. $(\mathbf{I}$ and K) Thirty days after bone marrow transplant with naphthalene injury. (I) Low-power image. (J and K) High-power image. Scale bars: 20 um. Original magnification, $\times 60\left(\mathbf{A}, \mathbf{B}, \mathbf{C}, \mathbf{F}\right.$, and I); $\times 90\left(\mathbf{D}, \mathbf{H}\right.$, and J); $\times 120(\mathbf{E}, \mathbf{G}, \mathbf{K})$. (L) Quantification of donor Ccsp ${ }^{+}$cells in the airways. Each bar represents mean \pm SEM ( $n=3$ per group). ${ }^{*} P<0.01$ compared with uninjured WT group; $\uparrow<0.05$ compared with uninjured WT group; $\ddagger P<0.001$ compared with injured WT group.

naphthalene. Naphthalene-injured and uninjured mice were then sacrificed 4 or 30 days following injury. Bone marrow of knockout mice that received wild-type marrow showed detectable levels of $\mathrm{Ccsp}^{+}(1.6 \%)$ cells after 30 days (Figure 5C). As in wild-type animals, the number of Ccsp ${ }^{+}$cells increased to over $4 \%$ at 4 days after naphthalene injury. In contrast, mice that received Ccsp knockout marrow showed no detectable $\mathrm{Ccsp}^{+}$cells in the marrow (Figure 5C). Western blotting for Ccsp showed detectable levels of Ccsp protein $(\sim 11 \mathrm{kDa})$ in the lungs and kidney but not the heart or liver of BM transplant recipients that received wild-type marrow (Figure 5D). Real-time PCR confirmed low but detectable levels of Ccsp mRNA in the lungs of mice that received Ccsp ${ }^{+}$cells (data not shown). Immunoreactive Ccsp was seen in the lungs of animals that received wild-type marrow or Ccsp knockout marrow supplemented with wild-type Ccsp ${ }^{+}$cells. Expression was weak and diffuse, spanning the majority of the airway epithelium (data not shown). Recipients that received Ccsp knockout marrow showed no positive staining for Ccsp. 

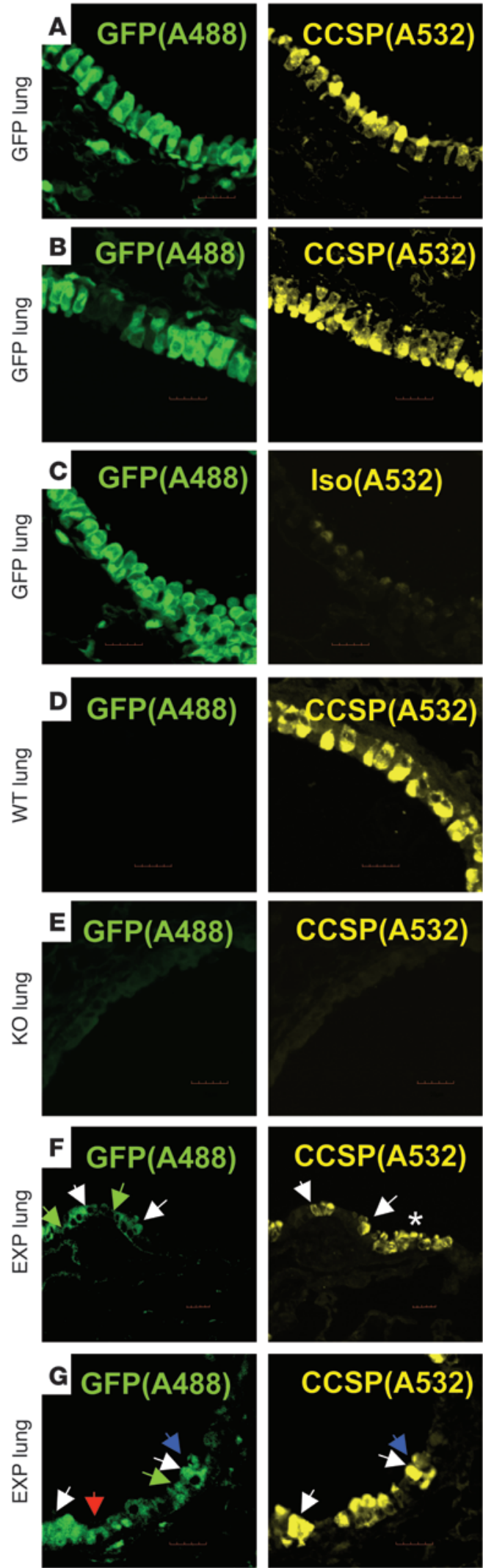
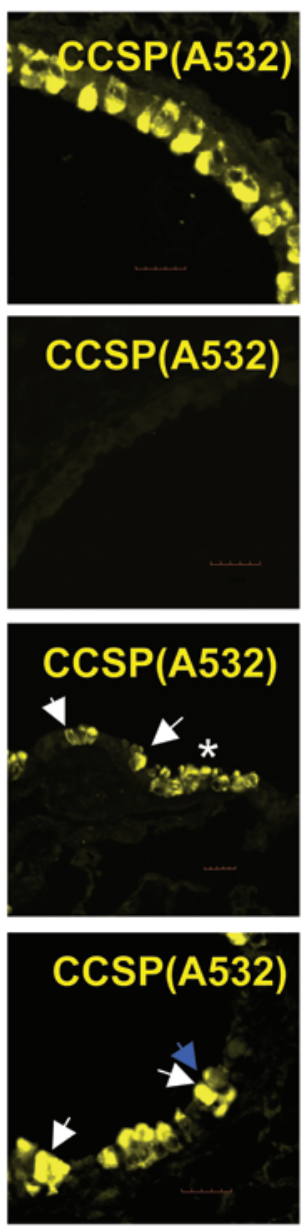
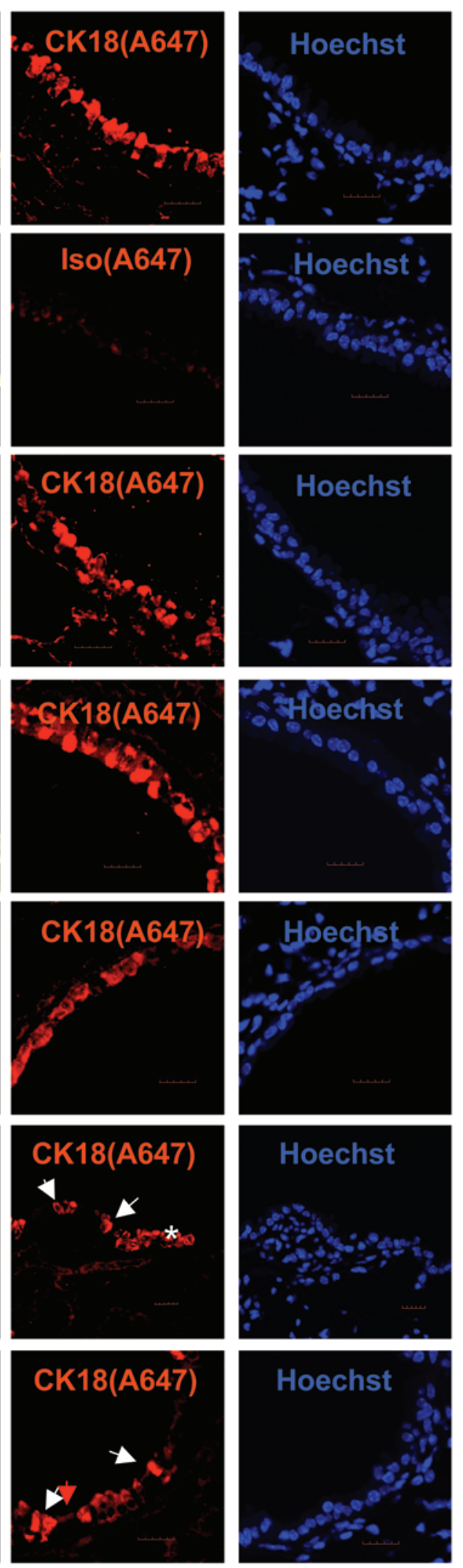
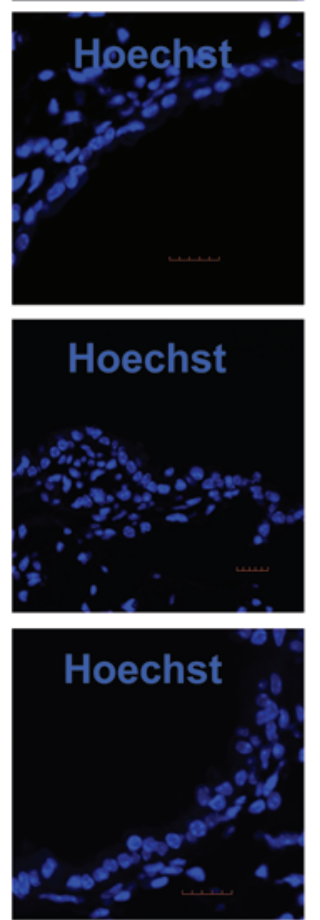

\section{Figure 7}

Airway-retained GFP ${ }^{+} \mathrm{Ccsp}^{+}$ donor cells express epithelialspecific K18 protein. (A-E) Staining controls for GFP, Ccsp, and K18. (F and $\mathbf{G}$ ) Representative experimental group in which Ccsp knockout recipients received bone marrow transplant of $\mathrm{Ccsp}+\mathrm{GFP}^{+}$ BMCs (EXP lung) and showed coexpression of GFP, Ccsp, and K18 under low-power magnification (F) and highpower magnification (G). White arrows point to $\mathrm{GFP}+{ }^{+} \mathrm{Csp}+\mathrm{K}^{+} 8^{+}$ cells, green arrows point to $\mathrm{GFP}^{+} \mathrm{Ccsp}^{-} \mathrm{K} 18^{-}$cells, red arrows point to $\mathrm{GFP}^{+} \mathrm{Ccsp}^{-}$ $\mathrm{K}^{1} 8^{+}$cells, blue arrows point to $\mathrm{GFP}^{+} \mathrm{Ccsp}^{+} \mathrm{K} 18^{-}$cells. Scale bars: $20 \mu \mathrm{m}$. Original magnification, $\times 60$. Asterisks indicate GFP-Ccsp+K18+ epithelium. $n=4$ per group. 
To determine whether and where donor $\mathrm{Ccsp}^{+} \mathrm{BMCs}$ were producing Ccsp in the lungs, transplants were repeated using donor $\mathrm{Ccsp}^{+}$cells from transgenic mice that constitutively expressed GFP under the $\beta$-actin promoter (BActGFP mice). Only $40 \%$ of BMCs expressed GFP, so these cells were sorted based on GFP and Ccsp expression. These donor cells also expressed the stem cell antigen 1 (Sca-1). Double immunofluorescence confocal microscopy for GFP (green) and Ccsp (red) of the lung from a BActGFP mouse showed colocalization of red and green fluorescence (Figure 6A). Isotype-matched (negative control) staining of the Ccsp knockout bone marrow transplant group is shown in Figure 6B. Wild-type recipients of $\mathrm{Ccsp}^{+} \mathrm{BMCs}$ showed infrequent donor-derived $\left(\mathrm{GFP}^{+}\right)$ $\mathrm{Ccsp}^{+}$cells with or without naphthalene lung injury. Most of the epithelium was either negative for donor cells (Figure 6C) or, when the cells were found, they did not express Ccsp (Figure 6D). An example of a Ccsp ${ }^{+}$donor-derived cell is shown in Figure 6E. In Ccsp knockout recipients however, donor-derived epithelial cells were easily found, even without naphthalene injury (Figure 6, F and G). Thirty days after airway injury in Ccsp knockout recipients, a much larger proportion of donor-derived $\mathrm{Ccsp}^{+}$cells were found in the airway epithelium (Figure 6, I and $\mathrm{K}$ ). In addition, $\mathrm{GFP}^{-}$also stained weakly positive for Ccsp protein (Figure $6, \mathrm{H}$ and J). Not all donor cells expressed Ccsp in the airway epithelium, even though all of the donor BMCs were $\mathrm{Ccsp}^{+}$at the time of marrow transplantation. Quantification of the extent to which the donor $\left(\mathrm{GFP}^{+} \mathrm{Ccsp}^{+}\right)$cells contributed to airway repopulation is shown in Figure 6L. Normal GFP mouse airway contains $86.01 \% \pm 8.18 \% \mathrm{GFP}^{+} \mathrm{Ccsp}^{+}$cells. Following bone marrow transplantation of $\mathrm{GFP}^{+} \mathrm{Ccsp}^{+} \mathrm{Sca}-1^{+}$cells into Ccsp knockout mice (without any additional airway injury), $56.25 \% \pm 21.60 \%$ of airway cells were $\mathrm{GFP}^{+} \mathrm{Ccsp}^{+}$cells at 32 days and $25.52 \% \pm 13.62 \%$ were $\mathrm{GFP}^{+} \mathrm{Ccsp}^{+}$at 62 days after bone marrow transplantation. Rare donor cells $(<0.5 \%)$ were found in the airways of wild-type recipients with or without naphthalene-induced airway injury.

To determine whether $\mathrm{GFP}^{+} \mathrm{Ccsp}^{+}$donor cells contributed to mature epithelium, triple immunofluorescence staining for GFP, Ccsp, and K18 was performed on the lung samples from the experiment described above (Figure 7). We found coexpression of $\mathrm{GFP}^{+} \mathrm{Ccsp}^{+} \mathrm{K}^{+} 8^{+}$in cells with appropriate localization and morphology similar to native epithelial cells, suggesting that the $\mathrm{Ccsp}^{+} \mathrm{BMCs}$ had contributed to a normal-looking epithelium. We also found $\mathrm{GFP}^{-} \mathrm{Ccsp}^{+} \mathrm{K}^{+} 8^{+}, \mathrm{GFP}^{+} \mathrm{Ccsp}^{-} \mathrm{K}^{-} 8^{-}, \mathrm{GFP}^{+} \mathrm{Ccsp}^{-} \mathrm{K}^{+} 8^{+}$, and $\mathrm{GFP}^{+} \mathrm{Ccsp}^{+} \mathrm{K}^{-} 8^{-}$cells. Since real-time PCR on sorted Ccsp ${ }^{+}$and $\mathrm{Ccsp}^{-}$cells showed no other epithelial gene expression such as K18, K5, or AQP5 (Supplemental Figure 5), we speculate that the in vivo detection of $\mathrm{Ccsp}^{+} \mathrm{K}_{18}{ }^{+}$donor cells was a result of phenotypic conversion possibly induced by the lung niche.

\section{Discussion}

Both human and mouse bone marrow contained rare cells that express an epithelial secretory protein, CCSP. Real-time PCR confirmed that these cells transcribe Ccsp. We were able to distinguish and isolate these cells by surface expression of Ccsp. Following airway injury, both marrow and blood $\mathrm{Ccsp}^{+}$cells increased. After culture, $\mathrm{Ccsp}^{+} \mathrm{BMCs}$ expressed proteins involved in important epithelial functions, including proSpc, CFTR, and ENaC, and exhibited sodium transport properties consistent with functional $\mathrm{ENaC}$. After culture at ALI, we were able to induce Ccsp ${ }^{+} \mathrm{BMCs}$ to differentiate into various epithelial cell lineages coexpressing markers of ciliated, type I, type II, and/or basal cells. Transtracheal or intravenous delivery of $\mathrm{Ccsp}^{+}$cells resulted in greater retention in or on the airway epithelium compared with a mixed population of bone marrow or Ccsp- cells. Bone marrow transplantation of $\mathrm{Ccsp}^{+} \mathrm{BMCs}$ into Ccsp knockout mice demonstrated airway Ccsp expression, probably due to both localized engraftment and absorption of the Ccsp protein secreted by Ccsp-expressing cells. Ccsp expression was restored at the protein and mRNA levels, although substantial airway repopulation was only observed in Ccsp knockout mice receiving Ccsp $\mathrm{p}^{+} \mathrm{BMCs}$, compared with mice with normal pulmonary expression of Ccsp.

Many reports have suggested a beneficial role of bone marrow stem cells in tissue regeneration (15-18). However, the specific subpopulations of BMCs that are involved remain elusive, especially for the lung. Reports of BMC-derived chimerism in the pulmonary epithelium remain controversial, as some studies suggest that the hematopoietic precursors contribute to the lung epithelium $(2,19)$, while others indicate a mesenchymal derivation (4, 20). Support for a hematopoietic contribution include studies by Krause et al. (2), who found long-term donor cell engraftment (up to $20 \%$ ) from highly purified cells (ELH cells) injected into lethally irradiated mice. On the other hand, a recent publication further supports the epithelial differentiation potential of mesenchymal stem cells derived from the cord blood (CB-MSCs). These cells, when cultured in media supporting lung epithelial cells, can be induced to express lung-specific markers including Ccsp, proSpc, and CFTR (21). Intravenous administration of cord blood MSCs into NOD-SCID mice following low-dose irradiation resulted in a low level of airway engraftment of the cord blood MSCs that expressed cytokeratin and CFTR. Our studies may help to reconcile these findings. The population described here expressed Ccsp, a marker shown to identify endogenous pulmonary progenitor and stem cells (10-12), while also expressing hematopoietic markers. These $\mathrm{Ccsp}^{+}$cells were found in the plastic-adherent fraction and expressed some markers used to characterize mesenchymal cells (22). However, CD90 and CD105 are also known to be expressed in $\mathrm{CD}_{4} 5^{+}$hematopoietic precursors $(23,24)$. Several reports have suggested that there is a developmental relationship between hematopoietic stem cells and their mesenchymal progenitors, in which nonadherent hematopoietic cells that can reconstitute the blood have also been shown to give rise to stromal cells $(25,26)$. Future work will need to be done to determine the relationship Ccsp ${ }^{+}$cells have with these early hematopoietic/mesenchymal precursors.

The relationship between $\mathrm{Cssp}^{+} \mathrm{BMCs}$ with other reported bone marrow-derived stem cells should be discussed. The Ccsp ${ }^{+}$cells identified here were largely $\mathrm{CD} 45^{+}$and were easily cultured in the absence of specialized media. This is in contrast to reports that have identified "early MSC progenitors" from plastic-adherent cultures grown in media enriched with growth factors that support the growth of the multipotent adult progenitor cells (MAPCs) (20). A recent study identified a novel primitive stage-specific embryonic antigen 1-positive $\left(\mathrm{SSEA} 1^{+}\right.$) MSC population that is capable of giving rise to different mesenchymal, endothelial, and hepatic cells in vivo (27). These SSEA $1^{+}$cells are co-isolated with the plastic-adherent mesenchymal stromal cells and are phenotypically Lin ${ }^{-} \mathrm{CD} 45^{-}$ CD $31-S c a-1^{+} \mathrm{CD} 105^{+} \mathrm{CD} 73^{+} \mathrm{CD} 44^{+}$vimentin ${ }^{+}$. Surprisingly, these cells can also give rise to $\mathrm{CD} 45^{+}$hematopoietic cells following marrow transplantation (27). While SSEA $1^{+}$cells appear to have multipotent stem cell characteristics, it remains to be elucidated whether culturing the cells in MAPC growth media (28) may have favored a multipotent phenotype. 
A previous report by Colter et al. identified a small $(\sim 7 \mu \mathrm{m})$ and agranular recycling stem cell (RS1) population within the MSC compartment that accounts for up to $30 \%$ of the 5 -day plasticadherent marrow cell population (29). Similar to $\mathrm{Ccsp}^{+}$cells, RS1 cells expressed CD90 and ckit but did not express CD45 and CD34. It also does not appear that the $\mathrm{Ccsp}^{+}$cells are the very small, embryonic-like (VSEL) stem cells identified by Kucia et al. (30). VSEL cells are much smaller $(2-4 \mu \mathrm{m})$ in size compared with $\mathrm{Ccsp}^{+}$cells and have pluripotent differentiation potential similar to embryonic stem cells. It may be possible that $\mathrm{Ccsp}^{+}$cells are derived from the VSEL cells. Future work will be done to identify early progenitors of the $\mathrm{Ccsp}^{+}$fraction and determine their relationship to VSEL cells, RS1 MSCs, SSEA ${ }^{+}$MSCs, and previously reported hematopoietic stem cells $(31,32)$.

The ability of a marrow cell to convert into cells with a lung phenotype in vivo may be associated with its cell cycle state. A recent publication demonstrated that $\mathrm{GFP}^{+} \mathrm{CD} 45^{-}$stromal cells induced to enter the $G_{1} / S$ phase of the cell cycle with specific cytokines (IL-3, IL-6, and IL-11) resulted in a 3-fold increase in conversion to $\mathrm{GFP}^{+}$cytokeratin ${ }^{+}$pulmonary epithelial cells and lung homing potential (33). The cell cycle state of the Ccsp $\mathrm{P}^{+}$ BMCs when cultured under ALI and during bone marrow transplantation is unknown. However, since $\mathrm{Ccsp}^{+}$cells can expand rapidly in vitro, it is possible that these cells are in the $G_{1} / S$ phase of the cell cycle. Future work will need to be done to determine the effect of cell cycle status on the differentiation potential of the $\mathrm{Ccsp}^{+}$population.

The presence of the Ccsp protein in Ccsp knockout lung epithelial cells negative for GFP following marrow reconstitution with $\mathrm{GFP}^{+} \mathrm{Ccsp}^{+}$cells has several possible explanations. A likely explanation is that the Ccsp protein itself may be transferred. Alternatively, genetic information may be transferred between cells in the radiation-injured lung. Quesenberry's group has shown such transfer from radiation-injured lung epithelial cells through microvesicles that subsequently are absorbed by marrow cells (34). It is possible that the converse may also be true, whereby genetic information, presumably at the RNA level, is released by $\operatorname{Cssp}^{+} \mathrm{BMCs}$ that can be absorbed by lung epithelial cells, thus restoring Ccsp expression. A third possibility is that the GFP transgenic lineage tracing system did not breed true. That is, that $\mathrm{GFP}^{+} \mathrm{BMCs}$ gave rise to $\mathrm{GFP}^{-}$progeny during differentiation. If this is the case, then the high numbers of $\mathrm{GFP}^{+}$seen in the airway are still an underestimate.

Bone marrow-derived cells have been suggested to give rise to lung epithelial cells in vitro $(7,35)$ and in vivo $(2,5,13)$. However, the precise fate of specific BMCs has not been well characterized to date. In vitro, culturing $\mathrm{Ccsp}^{+}$BMCs at ALI for 4 weeks to induce epithelial differentiation resulted in multilineage differentiation into numerous distinct epithelial cell types. $\mathrm{Ccsp}^{+}$ $\mathrm{BMCs}$ differentiated into $\mathrm{Ccsp}^{+}$, $\mathrm{proSpc}^{+}, \mathrm{AQP}^{+}, \mathrm{K}^{4} 4^{+}$, acetylated $\alpha$-tubulin ${ }^{+}$, and intermediary cells coexpressing different epithelial lineage markers such as $\operatorname{proSpc}^{+} \mathrm{AQP} 5^{+}$, acetylated $\alpha-$ tubulin ${ }^{+}$proSpc $^{+}$, and $\mathrm{Ccsp}^{+}$proSpc $^{+}$cells. Coexpression of Ccsp and proSpc suggests a resemblance to the recently described bronchoalveolar stem cell (BASC) (12). However, in addition to being CD34+, our Ccsp $\mathrm{P}^{+} \mathrm{BMCs}$ were $\mathrm{CD} 45^{+}$and $\mathrm{CD} 31^{+}$(data not shown), which makes them distinct from the BASC population. The full extent of differentiation possible using specific media or growth factors (36), coculture with epithelial cells (7), or differentiation in vivo was not tested, but these conditions might yield more differentiated epithelial cells.
Other studies have also suggested that the bone marrow may contain cells that already express epithelial or tissue-specific proteins. Gomperts et al. identified a K5-expressing cell in the marrow and peripheral blood that is recruited to the large airway epithelium following tracheal transplantation (37). The Ccsp ${ }^{+}$cells described here expressed $\mathrm{K} 5$ only after further culture suggesting $\mathrm{Ccsp}^{+} \mathrm{BMCs}$ may be a precursor for $\mathrm{K}^{+}$cells. Tissue-committed neural, cardiac, liver and muscle stem/progenitor cells have also been suggested to reside in the marrow (37-40). The data presented here support the notion that the marrow may contain many specific stem/progenitors cells that may be mobilized to its separate tissues upon injury.

Bone marrow $\mathrm{Ccsp}^{+}$cells may have substantial value as a source material for cell replacement therapies for epithelial diseases. Intrapulmonary stem cells that reside in distinct niches in the lung $(11,12,41-43)$ are considered the primary source of epithelial regeneration, but isolation and expansion of autologous cells from such sources is difficult and limits their practical utility. Bone marrow $\mathrm{Ccsp}^{+}$cells could be easily isolated and expanded. Unlike terminally differentiated lung $\operatorname{Ccsp}^{+}$cells, $\mathrm{Ccsp}^{+} \mathrm{BMCs}$ did not express the specific cytochrome $\mathrm{P} 450$ isoenzyme, CYP2F2 (14), which degrades naphthalene into toxic metabolites, and were not destroyed following naphthalene treatment. On the contrary, naphthalene increased the number of $\mathrm{Ccsp}^{+}$cells in the bone marrow and blood. This relative resistance to naphthalene injury is also seen in endogenous lung $\mathrm{Ccsp}^{+}$progenitor cells. In the bone marrow transplant experiments, far more marrow-derived cells were identified in the lungs when the recipient did not express Ccsp. In the presence of Ccsp protein (i.e. wild-type animals), marrow cells were only rarely identified in the lungs. We speculate that the Ccsp protein itself may be required for endogenous progenitor cell survival or proliferation, and in its absence, marrow-derived cells enjoy a competitive advantage. The Ccsp protein may be a rescue factor for endogenous and/or extrapulmonary progenitor cell survival and/or proliferation in the injurious milieu. Ccsp has been shown to be antiinflammatory and may regulate immune cells during injury $(44,45)$. Therefore, in the absence of the protein (as in Ccsp knockout recipients), endogenous progenitor cells may not be able to respond to lung injuries and therefore exogenous reparative cells such as $\mathrm{Ccsp}^{+} \mathrm{BMCs}$, which express their own Ccsp, may enjoy a competitive advantage.

Finally, while we can not rule out the possibility that cell fusion may occur in our model, this explanation seems unlikely because the number of $\mathrm{Ccsp}^{+}$donor cells that repopulated the airway epithelium of Ccsp knockout mice was quite high and studies including our own have suggested cell fusion events by BMCs are rare (9, 13). Harris et al. used a robust Cre-lox strategy to demonstrate that BMCs (containing hematopoietic cells and MSCs/progenitor cells) do not contribute to lung, liver, and skin epithelia by cell fusion with local epithelial cells (46). Since $\mathrm{Ccsp}^{+}$cells may also exist in the BMC fraction studied by Harris et al., it suggests cell fusion is unlikely an explanation for the higher level of donor cell repopulation we have observed.

$\mathrm{Ccsp}^{+} \mathrm{BMCs}$ have a greater propensity to engraft in the lung and, at least in vitro, have multiepithelial differentiation potential, suggesting that therapeutic delivery of $\mathrm{Ccsp}^{+}$cells may promote lung epithelial regeneration. Moreover, even with incomplete replacement of the epithelium, these cells restore Ccsp protein expression in the lung, which may decrease lung injury. 


\section{Methods}

Animals. Adult male (4 to 6 weeks old) C57BL/6, CD1 (Charles River Laboratories), $\beta$-actin-GFP (C57BL/ 6 mice constitutively expressing GFP under the control of the $\beta$-actin promoter $B A c t G F P$; The Jackson Laboratory) mice were used as BMC donors. Age- and strain-matched C57BL/6 and Ccsp knockout (47) female mice, respectively, were provided by Barry Stripp (Duke University, Durham, North Carolina, USA) and used as recipients. All animal procedures were approved by the University Health Network Animal Care Committee (Toronto, Ontario, Canada). All mice received care in compliance with the Principles of Laboratory Animal Care formulated by the National Society for Medical Research and the Guide for the Care and Use of Experimental Animals formulated by the Canadian Council on Animal Care. Human samples from volunteer healthy donors were collected under a protocol approved by University Health Network Research Ethics Board. All volunteers provided informed consent before participation in the study.

Animal procedures. Mouse whole bone marrow was harvested and cultured as previously described (13). Cells were cultured in DMEM (Invitrogen) supplemented with 10\% FBS (Invitrogen) and 1\% penicillin-streptomycin (Invitrogen). For lung injury studies, mice were given intraperitoneal injections of naphthalene (>99\% pure; Sigma-Aldrich), $200 \mathrm{mg}$ per kg body weight as previously described (13). For marrow transplantation studies, female Ccsp knockout or wild-type age-matched recipient mice were irradiated with $9.5 \mathrm{cGy}$. Within 2 hours of irradiation, female Ccsp knockout mice received cells via tail vein injection.

Fluorescence activated cell sorting and cell labeling. Freshly isolated BMCs and cultured, plastic-adherent BMCs from naphthalene-injured and noninjured mice were stained with rabbit anti-mouse Ccsp (1:1,000; Upstate Labs) followed by Alexa Fluor 488 secondary IgG (1:500; Invitrogen).

Real-time PCR analysis. Real-time PCR (SYBR green detection method; Applied Biosystems) was performed for amplification of the genes (Supplemental Methods). To quantify the number of BMCs retained in the lung, genomic DNA (gDNA) from the left lung of each mouse was extracted as previously described (13). GAPDH was determined to be the most stable housekeeping gene by the geNorm program; therefore, it was used as the housekeeping gene to normalize gene expression levels using REST-384 program (a relative expression software tool; ref. 48; geNorm and REST384 programs available at http://download.gene-quantification.info). Normalized mRNA or gDNA levels are expressed as relative to the positive control samples (airway epithelial cells or male lung cells).

Immunofluorescence. For assessment of epithelial marker expression, immunofluorescence staining for proSpc (AB3428; Chemicon; or H-00006440-M01, monoclonal version from Abnova); K18 (clone Ks 18.04);
K14 (Vector Labs); CD34 (553732; BD Pharmingen); CD45-PE (clone 30F11; BD Biosciences - Pharmingen); collagen type IV, vimentin, CD90, CD105, CD106, and CD73-PE (all from BD); collagen type I (Rockland); CFTR (MAB1660; R\&D Systems); ENaC (AB3532P; Chemicon); acetylated $\alpha$-tubulin IV (ab24610; Abcam); and AQP5 (178615; Calbiochem) was performed as previously described (13).

Epithelial differentiation assay. For ALI cultures, to determine whether Ccsp ${ }^{+}$ cells could give rise to other epithelial cell lineages, plastic-adherent BMCs were sorted into $\mathrm{Ccsp}^{+}$and $\mathrm{Ccsp}^{-}$cells after 7 days in culture and cultured under ALI for an additional 4 weeks using a slightly modified protocol that has been previously described (ref. 49; modifications are described in Supplemental Methods).

Additional methods are provided in Supplemental Methods.

Statistics. Data are presented as mean \pm SEM. For comparison of freshly isolated BMCs and cultured plastic-adherent BMCs phenotype, 1-way ANOVA followed by Tukey's post-hoc tests (using GraphPad Prism 4.0) were performed. For patch clamp analysis, means were compared with Student's paired 2-tailed $t$ test where appropriate using the SigmaStat software (Jandal Scientific Co). Statistical significance was defined as $P<0.05$.

\section{Acknowledgments}

Special thanks to Barry Stripp for the Ccsp knockout animals, goat anti-Ccsp antibody, and many helpful discussions. The authors also thank Masashi Gotoh for his technical support. Pilot work for the patch clamp analysis was performed with help from Hugh O'Brodovich at the Hospital for Sick Children, Toronto, Ontario, Canada. This work was supported by the Canadian Cystic Fibrosis Foundation and the Canadian Institutes of Health Research (CIHR). A. Keating holds the Gloria and Seymour Epstein Chair in Cell Therapy and Transplantation at University Health Network (University of Toronto). T.K. Waddell is supported by a CIHR Scientist Award and the R. Fraser Elliott Chair in Transplantation Research. A.P. Wong received a Studentship Award from the Canadian Cystic Fibrosis Foundation.

Received for publication July 22, 2008, and accepted in revised form December 3, 2008.

Address correspondence to: Thomas Waddell, Toronto General Hospital, 9N-949, 200 Elizabeth Street, Toronto, Ontario, Canada M5G 2C4. Phone: (416) 340-3432; Fax: (416) 340-4556; E-mail: tom.waddell@uhn.on.ca.
1. Kotton, D.N., et al. 2001. Bone marrow-derived cells as progenitors of lung alveolar epithelium. Development. 128:5181-5188.

2. Krause, D.S., et al. 2001. Multi-organ, multi-lineage engraftment by a single bone marrow-derived stem cell. Cell. 105:369-377.

3. Macpherson, H., et al. 2005. Bone marrow-derived SP cells can contribute to the respiratory tract of mice in vivo. J. Cell Sci. 118:2441-2450.

4. Ortiz, L.A., et al. 2007. Interleukin 1 receptor antagonist mediates the antiinflammatory and antifibrotic effect of mesenchymal stem cells during lung injury. Proc. Natl. Acad. Sci. U. S. A. 104:11002-11007.

5. Rojas, M., et al. 2005. Bone marrow-derived mesenchymal stem cells in repair of the injured lung. Am. J. Respir. Cell Mol. Biol. 33:145-152.

6. Xu, J., et al. 2007. Prevention of endotoxin-induced systemic response by bone marrow-derived mesenchymal stem cells in mice. Am. J. Physiol. Lung Cell Mol. Physiol. 293:L131-L141.

7. Wang, G., et al. 2005. Adult stem cells from bone marrow stroma differentiate into airway epithelial cells: potential therapy for cystic fibrosis. Proc. Natl. Acad. Sci. U. S. A. 102:186-191.

8. Kotton, D.N., Fabian, A.J., and Mulligan, R.C. 2005 Failure of bone marrow to reconstitute lung epithelium. Am. J. Respir. Cell Mol. Biol. 33:328-334.

9. Loi, R., Beckett, T., Goncz, K.K., Suratt, B.T., and Weiss, D.J. 2006. Limited restoration of cystic fibrosis lung epithelium in vivo with adult bone marrow-derived cells. Am. J. Respir. Crit. Care Med. 173:171-179.

10. Giangreco, A., Reynolds, S.D., and Stripp, B.R. 2002. Terminal bronchioles harbor a unique airway stem cell population that localizes to the bronchoalveolar duct junction. Am. J. Pathol. 161:173-182.

11. Hong, K.U., Reynolds, S.D., Giangreco, A., Hurley, C.M., and Stripp, B.R. 2001. Clara cell secretory protein-expressing cells of the airway neuroepithelial body microenvironment include a label-retaining subset and are critical for epithelial renewal after progenitor cell depletion. Am. J. Respir. Cell Mol. Biol. 24:671-681.

12. Kim, C.F., et al. 2005. Identification of bronchioal- veolar stem cells in normal lung and lung cancer. Cell. 121:823-835.

13. Wong, A.P., et al. 2007. Targeted cell replacement with bone marrow cells for airway epithelial regeneration. Am. J. Physiol. Lung Cell Mol. Physiol. 293:L740-L752.

14. Stripp, B.R., Maxson, K., Mera, R., and Singh, G. 1995. Plasticity of airway cell proliferation and gene expression after acute naphthalene injury. Am. J. Physiol. 269:L791-L799.

15. Horwitz, E.M., et al. 1999. Transplantability and therapeutic effects of bone marrow-derived mesenchymal cells in children with osteogenesis imperfecta. Nat. Med. 5:309-313.

16. Kocher, A.A., et al. 2001. Neovascularization of ischemic myocardium by human bone-marrowderived angioblasts prevents cardiomyocyte apoptosis, reduces remodeling and improves cardiac function. Nat. Med. 7:430-436.

17. Okamoto, R., et al. 2002. Damaged epithelia regenerated by bone marrow-derived cells in the human gastrointestinal tract. Nat. Med. 8:1011-1017. 
18. Otani, A., Kinder, K., Ewalt, K., Otero, F.J., Schimmel, P., and Friedlander, M. 2002. Bone marrowderived stem cells target retinal astrocytes and can promote or inhibit retinal angiogenesis. Nat. Med. 8:1004-1010.

19. Mattsson, J., Jansson, M., Wernerson, A., and Hassan, M. 2004. Lung epithelial cells and type II pneumocytes of donor origin after allogeneic hematopoietic stem cell transplantation. Transplantation. 78:154-157.

20. Jiang, Y., et al. 2002. Pluripotency of mesenchymal stem cells derived from adult marrow. Nature. 418:41-49.

21. Sueblinvong, V., et al. 2008. Derivation of lung epithelium from human cord blood-derived mesenchymal stem cells. Am. J. Respir. Crit. Care. Med. 177:701-711.

22. Horwitz, E.M., et al. 2005. Clarification of the nomenclature for MSC: the International Society for Cellular Therapy position statement. Cytotherapy. 7:393-395.

23. Sumikuma, T., et al. 2002. CD34+/CD90+ cells infused best predict late haematopoietic reconstitution following autologous peripheral blood stem cell transplantation. Br. J. Haematol. 117:238-244.

24. Pierelli, L., et al. 2001. CD105 (endoglin) expression on hematopoietic stem/progenitor cells. Leuk. Lymphoma. 42:1195-1206.

25. Olmsted-Davis, E.A., etal. 2003. Primitive adult hematopoietic stem cells can function as osteoblast precursors. Proc. Natl. Acad. Sci. U. S. A. 100:15877-15882.

26. Dominici, M., et al. 2004. Hematopoietic cells and osteoblasts are derived from a common marrow progenitor after bone marrow transplantation. Proc. Natl. Acad. Sci. U. S. A. 101:11761-11766.

27. Anjos-Afonso, F., and Bonnet, D. 2007. Nonhematopoietic/endothelial SSEA-1+ cells define the most primitive progenitors in the adult murine bone marrow mesenchymal compartment. Blood. 109:1298-1306.

28. Jiang, Y., et al. 2002. Multipotent progenitor cells can be isolated from postnatal murine bone marrow, muscle, and brain. Exp. Hematol. 30:896-904.
29. Colter, D.C., Class, R., DiGirolamo, C.M., and Prockop, D.J. 2000. Rapid expansion of recycling stem cells in cultures of plastic-adherent cells from human bone marrow. Proc. Natl. Acad. Sci. U.S.A 97:3213-3218.

30. Kucia, M., et al. 2006. A population of very small embryonic-like (VSEL) CXCR4(+)SSEA-1(+)Oct$4(+)$ stem cells identified in adult bone marrow. Leukemia. 20:857-869.

31. Zhong, J.F., et al. 2005. Gene expression profile of murine long-term reconstituting vs. short-term reconstituting hematopoietic stem cells. Proc. Natl. Acad. Sci. U. S. A. 102:2448-2453.

32. Yang, L., et al. 2005. Identification of Lin(-)Sca1( +)kit(+)CD34(+)Flt3- short-term hematopoietic stem cells capable of rapidly reconstituting and rescuing myeloablated transplant recipients. Blood. 105:2717-2723.

33. Dooner, M.S., et al. 2008. Conversion potential of marrow cells into lung cells fluctuates with cytokineinduced cell cycle. Stem Cells Dev. 17:207-219.

34. Aliotta, J.M., et al. 2007. Alteration of marrow cell gene expression, protein production, and engraftment into lung by lung-derived microvesicles: a novel mechanism for phenotype modulation. Stem Cells. 25:2245-2256.

35. Spees, J.L., et al. 2003. Differentiation, cell fusion, and nuclear fusion during ex vivo repair of epithelium by human adult stem cells from bone marrow stroma. Proc. Natl. Acad. Sci. U. S. A. 100:2397-2402.

36. Ali, N.N., et al. 2002. Derivation of type II alveolar epithelial cells from murine embryonic stem cells. Tissue Eng. 8:541-550.

37. Gomperts, B.N., et al. 2006. Circulating progenitor epithelial cells traffic via CXCR4/CXCL12 in response to airway injury. J. Immunol. 176:1916-1927.

38. Kucia, M., Ratajczak, J., Reca, R., Janowska-Wieczorek, A., and Ratajczak, M.Z. 2004. Tissue-specific muscle, neural and liver stem/progenitor cells reside in the bone marrow, respond to an SDF-1 gradient and are mobilized into peripheral blood during stress and tissue injury. Blood Cells Mol. Dis. 32:52-57.

39. Kucia, M., et al. 2006. Cells enriched in markers of neural tissue-committed stem cells reside in the bone marrow and are mobilized into the peripheral blood following stroke. Lenkemia. 20:18-28.

40. Kucia, M., et al. 2004. Cells expressing early cardiac markers reside in the bone marrow and are mobilized into the peripheral blood after myocardial infarction. Circ. Res. 95:1191-1199.

41. Hong, K.U., Reynolds, S.D., Watkins, S., Fuchs, E., and Stripp, B.R. 2004. Basal cells are a multipotent progenitor capable of renewing the bronchial epithelium. Am. J. Pathol. 164:577-588.

42. Reynolds, S.D., et al. 2007. Molecular and functional properties of lung SP cells. Am. J. Physiol. Lung Cell Mol. Physiol. 292:L972-L983.

43. Summer, R., et al. 2003. Side population cells and Bcrp1 expression in lung. Am. J. Physiol. Lung Cell Mol. Physiol. 285:L97-L104.

44. Harrod, K.S., Mounday, A.D., Stripp, B.R., and Whitsett, J.A. 1998. Clara cell secretory protein decreases lung inflammation after acute virus infection. Am. J. Physiol. 275:L924-L930.

45. Hayashida, S., Harrod, K.S., and Whitsett, J.A. 2000. Regulation and function of CCSP during pulmonary Pseudomonas aeruginosa infection in vivo. Am. J. Physiol Lung Cell Mol. Physiol. 279:L452-L459.

46. Harris, R.G., et al. 2004. Lack of a fusion requirement for development of bone marrow-derived epithelia. Science. 305:90-93.

47. Stripp, B.R., Reynolds, S.D., Plopper, C.G., Boe, I.M., and Lund, J. 2000. Pulmonary phenotype of CCSP/UG deficient mice: a consequence of CCSP deficiency or altered Clara cell function? Ann. N. Y. Acad. Sci. 923:202-209.

48. Pfaffl, M.W., Horgan, G.W., and Dempfle, L. 2002. Relative expression software tool (REST) for groupwise comparison and statistical analysis of relative expression results in real-time PCR. Nucleic Acids Res. 30:e36.

49. You, Y., Richer, E.J., Huang, T., and Brody, S.L. 2002. Growth and differentiation of mouse tracheal epithelial cells: selection of a proliferative population. Am. J. Physiol. Lung Cell Mol. Physiol. 283:L1315-L1321. 